\title{
A Conjecture on Einstein, the Independent Reality of Spacetime Coordinate Systems and the Disaster of 1913
}

\author{
John D. Norton \\ Department of History and Philosophy of Science \\ University of Pittsburgh, Pittsburgh PA 15260 \\ jdnorton+@ pitt.edu
}

Preprint of "A Conjecture on Einstein, the Independent Reality of Spacetime Coordinate Systems and the Disaster of 1913," pp. 67-102 in A. J. Kox and J. Einsenstaedt, eds., The Universe of General Relativity. Einstein Studies

Volume 11. Boston: BirkhŠuser, 2005.

Two fundamental errors led Einstein to reject generally covariant gravitational field equations for over two years as he was developing his general theory of relativity. The first is well known in the literature. It was the presumption that weak, static gravitational fields must be spatially flat and a corresponding assumption about his weak field equations. I conjecture that a second hitherto unrecognized error also defeated Einstein's efforts. The same error, months later, allowed the hole argument to convince Einstein that all generally covariant gravitational field equations would be physically uninteresting. 


\section{Introduction}

This paper will present elementary accounts of both errors described

above. The first will be reviewed in Sections 2 and 3. The second, the new conjecture, will be motivated in Section 4, the hole argument sketched in relevant detail in Section 5 and the conjecture itself developed in Section 6. Conclusions are in Section 7.

By mid 1913, Einstein had come so close. He had the general theory of relativity in all its essential elements. This theory, he believed, would realize his ambition of generalizing the principle of relativity to acceleration. It would harbor no preferred coordinate systems and its equations would remain unchanged under arbitrary coordinate transformation; that is, they would be generally covariant. Yet, in spite of the able mathematical assistance of his friend Marcel Grossmann, this vision of general covariance was slipping away. The trouble lay in his gravitational field equations. He had considered what later proved to be the equations selected in November 1915 for the final theory, at least in the source free case. But he had judged them wanting and could find no generally covariant substitute. So in his joint "Entwurf" paper with Marcel Grossmann, ${ }^{1}$ Einstein published gravitational field equations of unknown and probably very limited covariance. This was the disaster of 1913 . Nearly three dark years lay ahead for Einstein as he struggled to satisfy himself that these unnatural equations were well chosen. Towards the end of 1915, a despairing and exhausted Einstein returned to general covariance and ultimately to the gravitational field equations that now bear his name.

What had gone wrong? How did Einstein manage to talk himself out of these final equations for nearly three years? Historical scholarship of the last two decades has given us a quite detailed answer to these questions. ${ }^{2}$

\footnotetext{
${ }^{1}$ Einstein and Grossmann, 1913.

${ }^{2}$ For an entry into this extensive literature see Stachel (1980), Norton (1983), (1984), (1986), Earman and Janssen (1993), Howard and Norton (1993), Editorial Notes in Klein et al. (1995), Janssen (1999), Renn and Sauer (1996), (1999).
} 
Much of this answer comes from Einstein's "Zurich Notebook,"3 a notebook of private calculations that catalogs Einstein's deliberations from his early acquaintance with the new mathematical methods required by his theory, through the evaluation of candidate gravitational field equations to the derivation of the gravitational field equations of the 1913 "Entwurf" theory. One error has long been understood. Whatever else the theory may do, it must return Newton's theory of gravitation in the domain of weak, static fields in which that older theory has been massively confirmed. Einstein made some natural but erroneous assumptions about weak static fields and the corresponding form his gravitational field equations must take in the weak field limit. They made recovery of this Newtonian limit impossible from the natural gravitational field equations.

This error alone does not suffice to explain fully Einstein's misadventure of 1913. For he proved able to find gravitational field equations that were both of very broad covariance and also satisfied his overly restrictive demands for weak, static fields. These equations too were developed in the Zurich Notebook but rejected in 1913 without clear explanation. Einstein must have later judged that rejection hasty, for these same equations were revived and endorsed in a publication of early November 1915 (Einstein 1915) when he returned to general covariance. What explains his 1913 rejection of these equations? What had he found by November 1915 that now made them admissible? Some additional error must explain it.

The problem has been investigated in detail by a research group to which I belong. ${ }^{4}$ Several possible explanations have been found. Some are related to hitherto unnoticed idiosyncrasies in Einstein's treatment of

\footnotetext{
${ }^{3}$ Presented with commentary and annotation as Document 10 in Papers, Vol. 4. and in Renn, Sauer et al.(forthcoming).

${ }^{4}$ The group was founded in 1991 under the direction of Peter Damerow and Jürgen Renn as the Working Group Albert Einstein, funded by the Senate of Berlin and affiliated with the Center for Development and Socialization, headed by Wolfgang Edelstein at the Max Planck Institute for Human Development in Berlin. It was continued after 1995 under the direction of Jürgen Renn as part of the project of studies of the integration and disintegration of knowledge in modern science at the Max Planck Institute for the History of Science in Berlin. Its members include
} 
coordinate systems when he developed the "Entwurf" theory. My purpose in this paper is to review one of these explanations that I believe will be of special interest to philosophers of space and time. The suggestion is that Einstein was misled and defeated by a fundamental conceptual error concerning the ontology of spacetime coordinate systems that lay hidden tacitly in his manipulations. What makes the account especially attractive is that we need attribute no new error to Einstein. It can be explained by the one other major error from this time that Einstein later freely conceded. That was his "hole argument," the vehicle that he would use repeatedly over the next three years to justify his abandoning of general covariance. By his own later analysis, the error of this argument was that Einstein accorded an existence to spacetime coordinate systems independent of the fields defined on them. While the earliest extant mention of the hole argument comes in November 1913, months after the completion of the "Entwurf" paper, I maintain that the error at its core had already corrupted Einstein's earlier attempts to recover the Newtonian limit from his candidate gravitational field equations. To recover this limit, Einstein needed to restrict his theory to specialized coordinates. If we presume that Einstein treated these limiting coordinate systems in the same way as those of the hole argument months later, it turns out that they appear to have an absolute character that contradicts the extended principle of relativity whose realization was the goal of Einstein's theory.

Moreover, the view I conjecture Einstein took of these limiting spacetime coordinate systems effectively precluded his acceptance of virtually all generally covariant gravitational field equations. So the hole argument was not merely a clever afterthought designed to legitimate Einstein's prior failure to find generally covariant gravitational field equations. Rather, in best Einstein tradition, it encapsulated in the simplest and most vivid form the deeper obstacle that precluded Einstein's acceptance of generally covariant gravitational field equations.

In this paper I will not reconstruct the evidential case for these errors in all detail, with its strengths and weaknesses; that has already been done in Norton (forthcoming). Rather my purpose is to present a primer for those who want a simple, self contained account of how Einstein went wrong and are willing to cede to the citations a more detailed analysis of the extent to which the account can be supported by our historical source material. I will

Michel Janssen, John D. Norton, Jürgen Renn, Tilman Sauer and John Stachel who are the co-authors of Renn, Sauer et al.(forthcoming). I am grateful to all members of this group for their contributions to stimulating discussion of the material in this paper. 
try to explain in the simplest terms possible what these two errors were, why Einstein found them alluring and how they defeated his efforts to find acceptable, generally covariant gravitational field equations. In the decades following Einstein's work, our formulations of general relativity have become far more sophisticated mathematically and more geometrical in spirit. My account will adhere as closely as practical to Einstein's older methods and terminology, for that will keep us closer to Einstein's thought and render the errors in it more readily intelligible. 


\title{
2. The Spatial Flatness of Weak, Static Gravitational Fields
}

\author{
In 1913, Einstein presumed that in weak static fields, his new \\ gravitation theory must deliver Euclidean spaces. His final theory of \\ 1915 allows spatial geometry to differ from the Euclidean in first order \\ quantities even in this limiting case.
}

Einstein was induced to give up the natural generally covariant gravitational field equations for his

"Entwurf" theory by his attempt to relate his new theory with the theory it supersedes, Newtonian gravitation theory.

We can see the problem as it appeared to him in 1913 if we compare the two theories. The Newtonian theory of gravitation is based on representing a gravitational field by a single potential $\varphi$ spread over a Euclidean space. Einstein's "Entwurf" theory of 1913 and his final general theory of relativity were built around a quadratic differential form ${ }^{5}$

$$
d s^{2}=\sum_{\mu, v} g_{\mu v} d x_{\mu} d x_{v}
$$

where ds is the invariant interval between neighboring events with spacetime coordinates $x_{\mu}$ and $x_{\mu}+d x_{\mu}$. The coefficients of the metric tensor $g_{\mu \nu}$

$$
\left[\begin{array}{llll}
g_{11} & g_{12} & g_{13} & g_{14} \\
g_{21} & g_{22} & g_{23} & g_{24} \\
g_{31} & g_{32} & g_{33} & g_{34} \\
g_{41} & g_{42} & g_{43} & g_{44}
\end{array}\right]
$$

now represent the gravitational field as well as the geometry of spacetime. The one gravitational potential of Newtonian theory has been replaced by 16 coefficients. Since the metric tensor is symmetric, we have $\mathrm{g}_{\mu \nu}=\mathrm{g}_{\nu \mu}$, so that only ten of these coefficients can be set independently. But that is still nine more than in Newtonian theory.

Newtonian theory has enjoyed spectacular confirmation in its domain of application. So, when Einstein's new theory is restricted to this domain, it must return results indistinguishable from those of Newtonian theory.

\footnotetext{
5In 1913, Einstein and Grossmann did not use the summation convention in their publications. The indices $\mu$ and $v$ range over 1,2, 3 and 4 .
} 


\subsection{Weak, Static Gravitational Fields}

Weak gravitational fields differ in quantities of first order of smallness from a Minkowski metric. A static field may be naturally sliced into three dimensional spaces and admits observers that see its geometric properties as time independent.

Newtonian theory prevails in the domain of weak, static gravitational fields and the restriction to this domain appears simple.

-In a weak gravitational field, the metric tensor differs only in small quantities from the metric tensor of a Minkowski spacetime, the spacetime of special relativity. That is, there is a coordinate system in which the metric can be written as

$$
g_{\mu \nu}=\eta_{\mu v}+h_{\mu v}
$$

where the background Minkowski metric is

$$
\eta_{\mu v}=\left[\begin{array}{cccc}
-1 & 0 & 0 & 0 \\
0 & -1 & 0 & 0 \\
0 & 0 & -1 & 0 \\
0 & 0 & 0 & 1
\end{array}\right]
$$

and the weak field perturbation is

$$
\mathrm{h}_{\mu \nu}<<\eta_{\mu \nu}
$$

-If a gravitational field is static, then we can find a coordinate system in which the coefficients $g_{\mu \nu}$ of the metric tensor are not functions of the time coordinate $\mathrm{x}_{4}$ and the mixed time-space components of the metric vanish: $\mathrm{g}_{14}=\mathrm{g}_{41}=\mathrm{g}_{24}=\mathrm{g}_{42}=\mathrm{g}_{34}=\mathrm{g}_{43}=0$. The metric has the form

$$
\left[\begin{array}{llll}
g_{11} & g_{12} & g_{13} & 0 \\
g_{21} & g_{22} & g_{23} & 0 \\
g_{31} & g_{32} & g_{33} & 0 \\
0 & 0 & 0 & g_{44}
\end{array}\right]
$$

These algebraic requirements admit a simple geometric interpretation. If an observer's worldline coincides with a curve of constant $\mathrm{x}_{1}, \mathrm{x}_{2}, \mathrm{x}_{3}$, such as the $\mathrm{x}_{4}$ coordinate axis, then that observer will see the geometric properties of space as time independent. The vanishing of the time-space components of the metric tensor allow the spacetime to be divided naturally into a family of time indexed three dimensional spaces as shown in Figure 1. Assuming that the 
coordinate system covers the entire spacetime, each three dimensional space is chosen by fixing a constant value for the time coordinate $\mathrm{x}_{4}$; the coordinates $\mathrm{x}_{1}, \mathrm{x}_{2}$ and $\mathrm{x}_{3}$ are then the coordinates of the three dimensional space and the metric of the space is

$$
\left[\begin{array}{lll}
g_{11} & g_{12} & g_{13} \\
g_{21} & g_{22} & g_{23} \\
g_{31} & g_{32} & g_{33}
\end{array}\right]
$$

These spaces are orthogonal to the observer's world line. That is, a vector tangent to the observer's worldline, such as $\mathrm{T}_{\mu}=(0,0,0,1)$, will be orthogonal to a vector tangent to the three dimensional space, such as $\mathrm{X}_{\nu}=(1,0,0,0)$, since $\sum_{\mu, v} g_{\mu v} T_{\mu} X_{v}=0$.

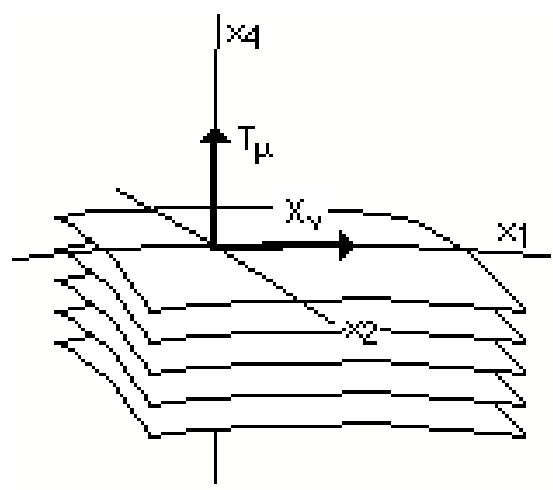

Figure 1. A Static Spacetime

\subsection{Recovering the Newtonian Limit}

As the Newtonian domain is approached, Einstein's new gravitation theory must restore Euclidean geometry in three dimensional space.

Einstein assumed that exact restoration occurs in weak, static fields since this reduces the ten coefficients of the metric tensor to the single potential of Newtonian theory.

These weak, static fields must now return the two properties characteristic of Newtonian gravitation theory: Euclidean space and a single gravitational potential. In the "Entwurf" paper Einstein presumed that this would happen in the simplest way imaginable. (Einstein and Grossmann, 1913, I §2) In quantities of first order of smallness, there would be just one component of $g_{\mu \nu}$ that was not constant. That would be $g_{44}$ which would represent the Newtonian gravitational potential $\varphi$. The components of the metric that return the geometry of the 
three dimensional spaces would be constants coinciding with Euclidean values. That is, in the relevant coordinate system, Einstein expected weak static fields to be of the form

$$
\begin{aligned}
& \text { Euclidean retric } \\
& \text { of space } \\
& {\left[\begin{array}{ccc:c}
-1 & 0 & 0 & 0 \\
0 & -1 & 0 & 0 \\
0 & 0 & -1 & 0 \\
\hdashline 0 & 0 & 0 & g_{4}=2
\end{array}\right]} \\
& \text { Newtonian } \\
& \text { gravitational } \\
& \text { potential }
\end{aligned}
$$

That the $\mathrm{g}_{44}$ corresponds to the Newtonian potential was strongly suggested to Einstein by the equations of motion of a slowly moving point mass in gravitational free fall in the theory. ${ }^{6}$ Such a point follows a geodesic in spacetime, a curve of extremal interval s. It is governed by the geodesic equation

$$
\frac{d^{2} x_{\mu}}{d s^{2}}+\sum_{\alpha \beta}\left\{\begin{array}{c}
\alpha \beta \\
\mu
\end{array}\right\} \frac{d x_{\alpha}}{d s} \frac{d x_{\beta}}{d s}=0
$$

where the Christoffel symbols are given as ${ }^{7}$

$$
\left\{\begin{array}{c}
\alpha \beta \\
\mu
\end{array}\right\}=\frac{1}{2} \sum_{v} \gamma_{\mu v}\left(g_{\alpha v, \beta}+g_{\beta v, \alpha}-g_{\alpha \beta, v}\right)
$$

Most terms in the geodesic equation vanish in quantities of the first order of smallness. In the Newtonian limit in this coordinate system, the derivatives $\frac{d x_{1}}{d s}, \frac{d x_{2}}{d s}, \frac{d x_{3}}{d s}$ correspond to the velocity of the mass and are thus each first order small. Thus the only significant component of the second term of the geodesic equation is the term in $\frac{d x_{4}}{d s} \frac{d x_{4}}{d s} \approx 1$. Because of the vanishing of the time-space components of the metric tensor, the related Christoffel symbols reduce to

$$
\left\{\begin{array}{c}
44 \\
i
\end{array}\right\}=\frac{1}{2} \sum_{v} \gamma_{i v}\left(g_{4 v, 4}+g_{4 v, 4}-g_{44, v}\right) \approx \frac{1}{2} g_{44, i}
$$

${ }^{6}$ For Einstein's abbreviated version of the calculation that follows, see Einstein $(1913, \S 8)$. He later explains to Michele Besso in a letter of December 21, 1915, that this result was misleading. (Papers, Vol. 8A, Doc. 168) 
for $\mathrm{i}=1,2,3$. The geodesic equation reduces to

$$
\frac{d^{2} x_{i}}{d s^{2}}=-\frac{1}{2} \frac{\partial g_{44}}{\partial x_{i}}
$$

The last of these equations shows that the $\mathrm{x}_{4}$ coordinate is linearly related to the interval along the mass' trajectory, justifying the interpretation of the $\mathrm{x}_{4}$ coordinate as time read by a clock--at least at this level of approximation--and the above assumption that $\frac{d x_{4}}{d s} \frac{d x_{4}}{d s} \approx 1$. The first equation relates the acceleration of the mass to the spatial gradient of $g_{44}$ exactly as in Newtonian theory

$$
\text { Acceleration }=-\operatorname{gradient}\left(\mathrm{g}_{44} / 2=\varphi\right)
$$

affirming the equation of the Newtonian potential $\varphi$ with $\mathrm{g}_{44} / 2$. Since the remaining coefficients of the metric play no role in this equation of motion, there seemed no obstacle to setting these to the constant Euclidean values.

\subsection{The Principle of Equivalence}

The principle of equivalence delivered Einstein one instance of a static gravitational field, a homogeneous gravitational field. That one instance proved to be spatially flat and Einstein readily generalized the result to all static fields.

Einstein had a stronger motivation for his conclusion that weak static fields are spatially flat. He had begun work on a relativistic theory of gravitation in Einstein (1907, Part V) with an ingenious idea he later called the "principle of equivalence." That principle supplied a heuristic means of generating a theory of gravitation. It began with one simple case. Einstein considered a Minkowski spacetime, the spacetime of special relativity, and determined how it would look to an observer in uniform acceleration. That observer would see all free objects uniformly accelerated in a direction opposite to that of the observer's acceleration. Since all these objects suffered the same acceleration, their motion conformed to a familiar characteristic of gravitation: all bodies fall alike, irrespective of their masses. It was as if the masses were under the influence of a homogeneous gravitational field.

${ }^{7}$ I continue to follow the notational conventions of Einstein and Grossmann's "Entwurf" paper. With the exception of the Christoffel symbols, all indices are written "downstairs". The contravariant form of the metric $\mathrm{g}_{\mu \nu}$ is written with the corresponding Greek letter as $\gamma_{\mu \nu}$. Commas denote coordinate differentiation. 
Einstein's principle of equivalence removes the "as if." It asserts the full equivalence of the two cases, a uniform acceleration in Minkowski spacetime and a homogenous gravitational field.

The principle of equivalence supplied Einstein with a relativistic account of one special case of the gravitational field, that of a homogeneous gravitational field. Einstein's development of a theory of static gravitational fields prior to 1913 (Einstein 1907, Part V; 1911; 1912a,b) resided in judiciously generalizing the properties of the homogeneous field to that of arbitrary static fields. For our purposes, the most important property of the homogeneous gravitational field produced by uniform acceleration was that its spatial geometry remained Euclidean. Therefore he assumed that spatial geometry in the presence of an arbitrary static field would also remain Euclidean ${ }^{8}$ and this presumption was carried over explicitly to the "Entwurf" theory.

The preservation of Euclidean geometry is seen most clearly if the transformation to uniform acceleration is analyzed within the framework of the "Entwurf" and later theories. We start with a Minkowski spacetime and a coordinate system $(\mathrm{X}, \mathrm{Y}, \mathrm{Z}, \mathrm{T})$ in which the expression for the interval is

$$
d s^{2}=-d X^{2}-d Y^{2}-d Z^{2}+d T^{2}
$$

We may represent a transformation from inertial to accelerated motion as a coordinate transformation following Einstein's usual practice. ${ }^{9}$ The simplest form of the transformation is given later in Einstein and Rosen (1935) as

$$
\mathrm{X}=\mathrm{x} \cosh \text { at } \quad \mathrm{Y}=\mathrm{y} \quad \mathrm{Z}=\mathrm{z} \quad \mathrm{T}=\mathrm{x} \sinh \text { at }
$$

where $\mathrm{a}$ is a constant that measures the magnitude of the acceleration. The expression for the interval transforms to

$$
d s^{2}=-d x^{2}-d x^{2}-d z^{2}+a^{2} x^{2} d t^{2}
$$

from which we recover an expression for the metric

\footnotetext{
${ }^{8}$ Einstein was aware in 1912 that the spatial geometry associated with acceleration need not be Euclidean. As he remarked in his (Einstein, 1912a, §1), the geometry fails to be Euclidean in the space association with uniform rotation. For further discussion of Einstein's use of the principle of equivalence, see Norton (1985).

${ }^{9}$ That is, the trajectories of reference bodies of the inertial frame are given by the timelike curves in spacetime picked out by constant values of the coordinates $\mathrm{X}, \mathrm{Y}$ and $\mathrm{Z}$. The trajectories of the reference bodies of the accelerated frame are given by the timelike curves in spacetime picked out by constant values of the coordinates $\mathrm{x}, \mathrm{y}$ and $\mathrm{z}$.
} 


$$
g_{\mu v}=\left[\begin{array}{cccc}
-1 & 0 & 0 & 0 \\
0 & -1 & 0 & 0 \\
0 & 0 & -1 & 0 \\
0 & 0 & 0 & a^{2} x^{2}
\end{array}\right]
$$

Even though this is not a case of a weak field, it is a static field and it conforms exactly to the expectations encoded in (4) that the spaces of such fields be Euclidean. ${ }^{10}$ The transformation (5) is shown graphically in Figure 2 and its reinterpretation as a homogeneous gravitational field in Figure 3.

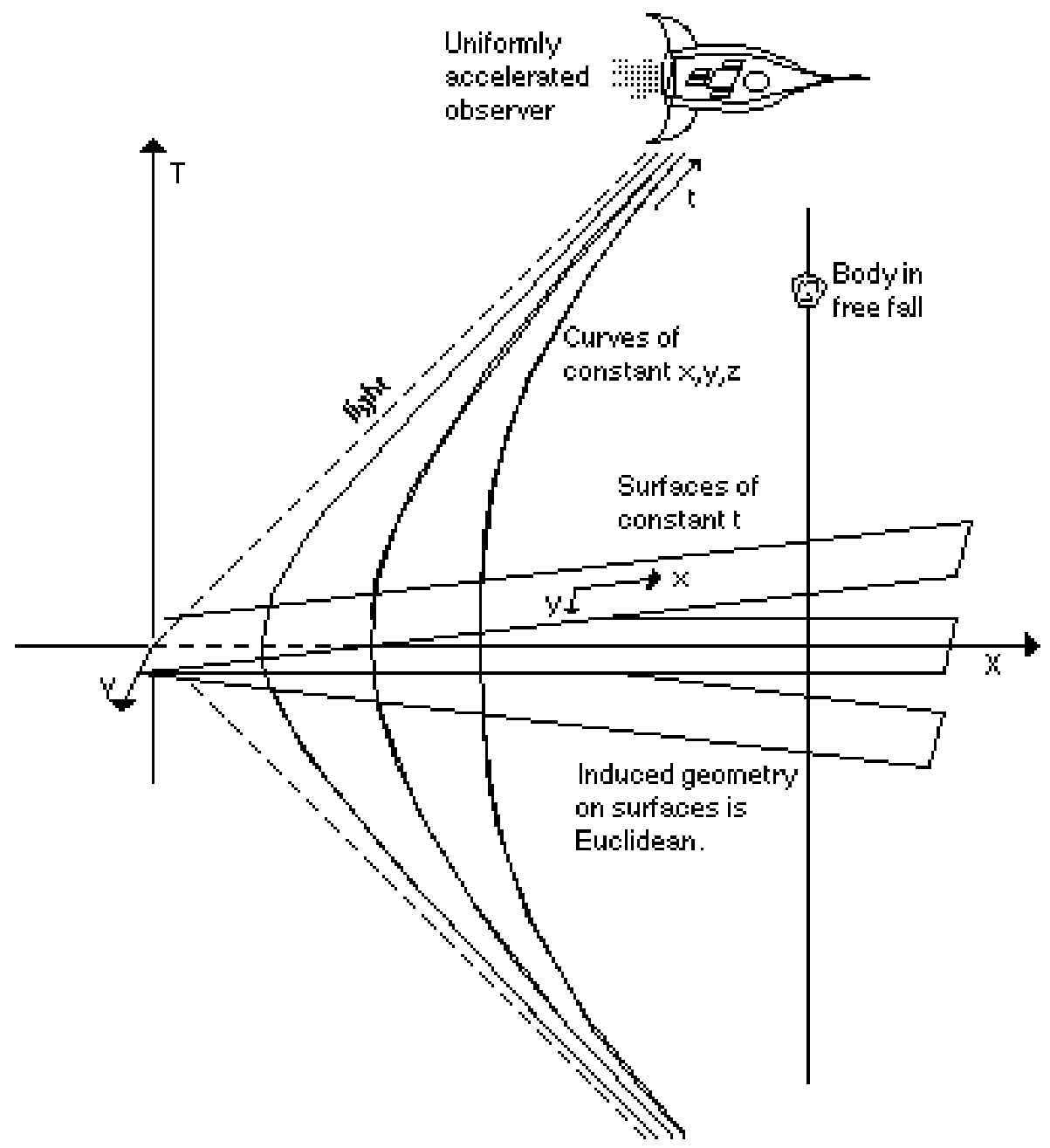

Figure 2. Principle of Equivalence: Uniform Acceleration in a Minkowski Spacetime...

${ }^{10}$ The term $\mathrm{g}_{44}=\mathrm{a}^{2} \mathrm{x}^{2}$ cannot be interpreted directly as a Newtonian potential since we are no longer dealing with the case of a weak field. 
Note that the transformation (5) does not cover the entire Minkowski spacetime but only one quadrant that lies outside the lightcone of the origin $\mathrm{X}=\mathrm{Y}=\mathrm{Z}=\mathrm{T}=0$.

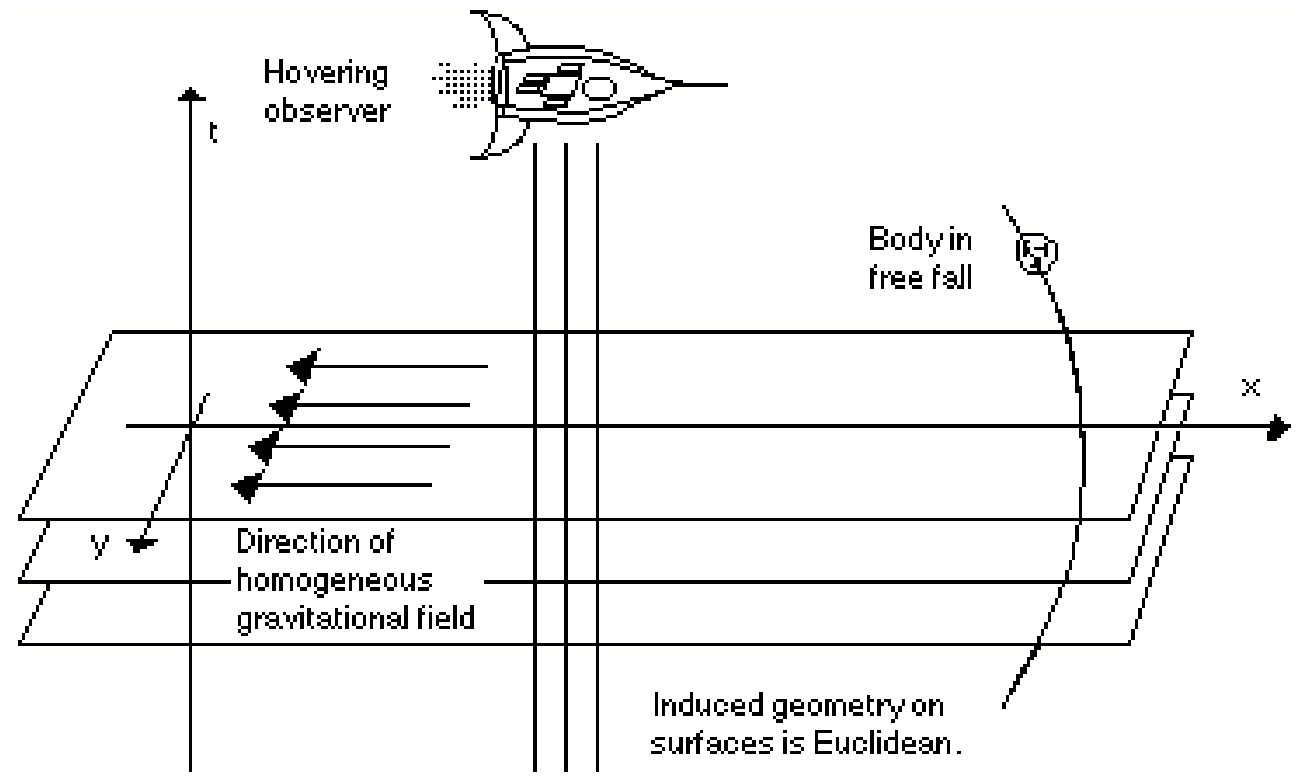

Figure 3 ... is Equivalent to a Homogeneous Gravitational Field

If we apply these expectations to one of the most important weak static fields addressed by the theory, the gravitational field of the sun, we recover Einstein's expectation that its metric tensor is

$$
g_{\mu v}=\left[\begin{array}{cccc}
-1 & 0 & 0 & 0 \\
0 & -1 & 0 & 0 \\
0 & 0 & -1 & 0 \\
0 & 0 & 0 & 1-\frac{\alpha}{r}
\end{array}\right]
$$

were $\alpha$ is determined by the mass of the sun and the coordinate $r$ is fixed as $r^{2}=x_{1}{ }^{2}+x_{2}^{2}+x_{3}{ }^{2}$.

\subsection{Contradiction with Einstein's Final Theory}

Weak, static gravitational fields are not spatially flat in Einstein's final theory of November 1915.

The modern reader will recognize immediately how seriously Einstein has strayed if this metric is compared with the exact solution for the gravitational field of the sun, the Schwarzschild solution. The three dimensional space surrounding the sun, even in weak field approximation, does deviate from Euclidean flatness. As Einstein would later ruefully discover, the metric tensor for the field of the sun in first order approximation is given by 


$$
g_{\mu \nu}=\left[\begin{array}{cccc}
-1-\frac{\alpha x_{1}^{2}}{r^{3}} & -\frac{\alpha x_{1} x_{2}}{r^{3}} & -\frac{\alpha x_{1} x_{3}}{r^{3}} & 0 \\
-\frac{\alpha x_{1} x_{2}}{r^{3}} & -1-\frac{\alpha x_{2}^{2}}{r^{3}} & -\frac{\alpha x_{2} x_{3}}{r^{3}} & 0 \\
-\frac{\alpha x_{1} x_{3}}{r^{3}} & -\frac{\alpha x_{2} x_{3}}{r^{3}} & -1-\frac{\alpha x_{3}^{2}}{r^{3}} & 0 \\
0 & 0 & 0 & 1-\frac{\alpha}{r}
\end{array}\right]
$$

this being the form Einstein published in Einstein (1915a) before the full expression for the Schwarzschild solution had been found. In the field of the sun, the three dimensional spaces orthogonal to the world tube of the sun are not Euclidean even at the level of first order quantities. That Einstein presumed otherwise would have disastrous consequences.

What Einstein expected in 1913:

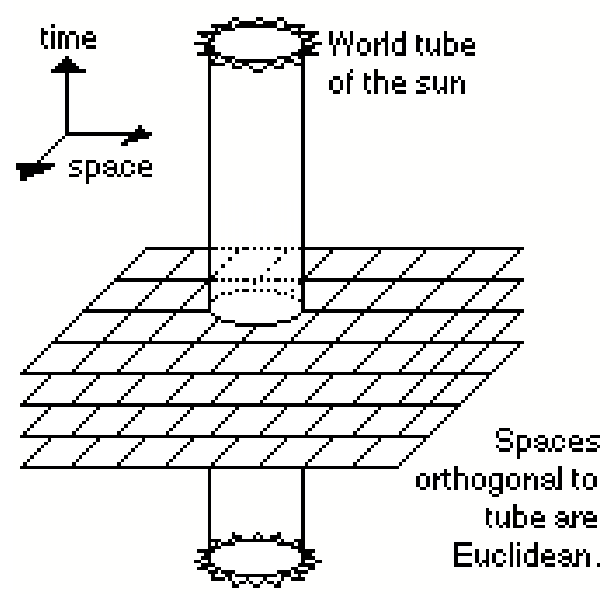

What Einstein realized in 1915 :

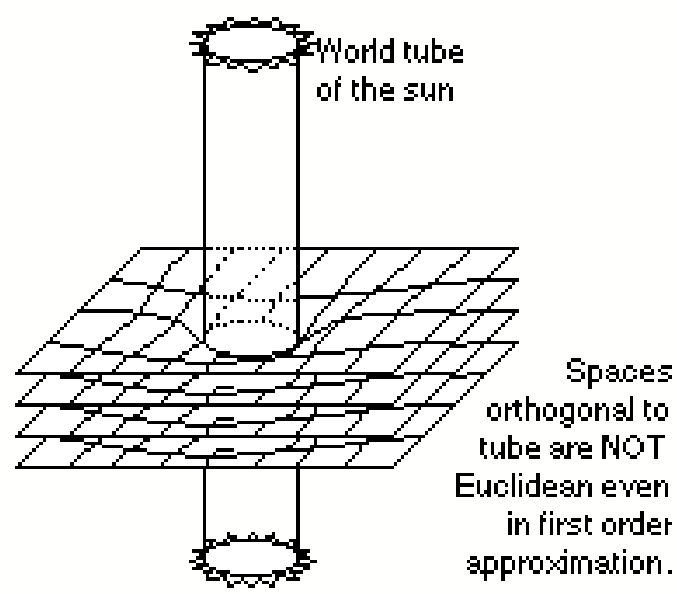

Figure 4 What Einstein Expected for the Gravitational Field of the Sun 


\title{
3. The Rejection of the Ricci Tensor
}

\author{
Einstein discarded the Ricci tensor as gravitation tensor since he could \\ find no coordinate condition that would reduce it to a spatially flat \\ Newtonian limit in the case of weak static fields.
}

Einstein expected weak, static gravitational fields to be spatially flat. Whether this would be so in his theory depends upon the gravitational fields the theory admits. That in turn is decided by the theory's gravitational field equations. In Newtonian theory, the single equation for the single potential $\varphi$ that selects the admissible gravitational fields is Poisson's equation

$$
\Delta \varphi=\left(\frac{\partial^{2}}{\partial x^{2}}+\frac{\partial^{2}}{\partial y^{2}}+\frac{\partial^{2}}{\partial z^{2}}\right) \varphi=4 \pi G \rho
$$

where $\mathrm{x}, \mathrm{y}$ and $\mathrm{z}$ are the Cartesian coordinates of space, $\mathrm{G}$ the gravitational constant and $\rho$ the density of matter.

Einstein sought a system of ten equations for the ten components of the metric tensor that would be the relativistic analog of this single equation. He expected it to have the form

$$
\mathrm{G}_{\mu \nu}=\mathrm{kT}_{\mu \nu}
$$

where $k$ is some constant, $T_{\mu \nu}$ is the stress energy tensor of matter and the gravitation tensor $G_{\mu \nu}$ is composed of terms in the metric tensor and its first and second derivatives. 


\title{
3.1 An Over-Simplified Form of the Gravitational Field Equations for the Weak Field
}

\author{
Einstein assumed a natural form (8) for the gravitational field \\ equations in weak field approximation that would return both Poisson's \\ equation of Newtonian theory and spatial flatness in simple cases.
}

Since Einstein's new theory must revert to Newton's in suitable limiting circumstances, Einstein's choice for gravitational field equations (7) must eventually revert to (6). To ensure this, Einstein presumed that his gravitational field equations (7) must first revert to the equations ${ }^{11}$

$$
\sum_{\alpha, \beta} \frac{\partial}{\partial x_{\alpha}}\left(\gamma_{\alpha \beta} \frac{\partial \gamma_{\mu \nu}}{\partial x_{\beta}}\right)+\left(\begin{array}{c}
\text { further terms } \\
\text { that vanish in the } \\
\text { first approximation }
\end{array}\right)=k T_{\mu v}
$$

in the case of a weak field (1). The motivation for this presumption is clear if one considers the form (8) takes in the weak, static field of (4) with a source of pressureless, motionless dust

$$
T_{\mu v}=\left[\begin{array}{llll}
0 & 0 & 0 & 0 \\
0 & 0 & 0 & 0 \\
0 & 0 & 0 & 0 \\
0 & 0 & 0 & \rho_{0}
\end{array}\right]
$$

where $\rho_{0}$ is the rest density of the matter. Equation (8) then reduces to

$$
\Delta \gamma_{44}=(-\mathrm{k}) \rho_{0}
$$

and for the remaining terms for which $\mu \neq 4$ or $v \neq 4$ (or both)

$$
\Delta \gamma_{\mu \nu}=0
$$

The first equation (8a) is merely the recovery of Poisson's equation (6) of Newtonian theory as expected. The second is readily solved in special cases to yield the result that the $\gamma_{\mu \nu}$ are constants whenever $\mu \neq 4$ or $v \neq 4$ so that the spatial

\footnotetext{
${ }^{11}$ Einstein and Grossmann (1913, I §5).An even simpler choice for the first term would have been $\sum_{\alpha . \beta} \gamma_{\alpha \beta} \frac{\partial^{2}}{\partial x_{\alpha} \partial x_{\beta}} \gamma_{\mu v}$. Einstein's choice of the term in (8) does not affect the outcome since the two agree in first
}

order quantities in the weak field. 
metric (3) is flat. This last conclusion affirmed Einstein's expectation that weak static fields are to be spatially flat; the same result is now recoverable from the natural equation (8)--another misleading corroboration of the result.

The special case for solving (8b) that Einstein considered in his Vienna lecture (Einstein, 1913, §8) was one in which the components of the metric tensor approached Minkowskian values at spatial infinity. Presumably he imagined the matter distribution $\rho_{0}$ to be concentrated into a central island of matter that diluted away completely with distance into an otherwise empty space, else the presumption of Minkowskian values at infinity would not be plausible. ${ }^{12}$

A weakness of Einstein's (1913) recovery of spatial flatness in the weak static field is that it depends on a source matter distribution with stress energy tensor (9). This is physically implausible since it is a matter distribution that does not undergo gravitational collapse but has no pressures or other stresses to counteract the collapse. Were the collapse not counteracted by such stresses, then the resulting velocity of the dust would contribute further non-zero terms (other than $\mathrm{T}_{44}$ ) to the stress energy tensor. If such stresses are present, then they would appear directly as further non-zero terms in the stress energy tensor. In either case, these new non-zero terms would defeat the derivation of $(8 b)$.

Einstein realized that his inference to spatial flatness was not quite so fragile. As he affirmed in a postcard to Erwin Freundlich of March 19, 1915 (Papers, Vol.8A, Doc. 63), spatial flatness could still be recovered for the space outside the sun if one assumed that the static mass distribution of the sun was sustained by pressures or stresses. For this case, the individual components of the stress energy tensor $T_{i k}(i, k=1,2,3)$ will in general be nonvanishing, so that ( $8 b)$ must be replaced by

\footnotetext{
${ }^{12}$ To ensure spatial flatness, Einstein does need an assumption of comparable strength. With it, the result of spatial flatness everywhere is quickly recovered. If Laplace's equation $\Delta \Psi=0$ holds everywhere in a sphere of radius $\mathrm{R}$, then a lemma asserts that the value of $\Psi$ at the center is just the integrated average of the values of $\Psi$ on the sphere's surface. Pick some arbitrary point in space and consider a family of spheres centered on it that extend to spatial infinity. If $\Psi$ is to approach the same constant value $\Psi_{\infty}$ in all directions at spatial infinity, then we must have $\Psi=\Psi_{\infty}$ at the center if the lemma is to hold for all the spheres. Replace $\Psi$ successively by each value of $\gamma_{\mu \nu}$ in (8b) and we conclude that each has Minkowskian values throughout the spacetime.
} 


$$
\Delta \gamma_{\mathrm{ik}}=(-\mathrm{k}) \mathrm{T}_{\mathrm{ik}}
$$

But, as Einstein noted, the condition of equilibrium entailed the vanishing of the integrals over space $\int T_{i k} d V=0$, a result due also to Laue, as Einstein also noted. (For more on Laue's analysis, see Norton, 1992, §9.) This in turn entailed that the coefficients $\gamma_{\mathrm{ik}}$ adopt Minkowskian values under suitable conditions. While Einstein did not complete the proof, it is easy if we presume spherical symmetry for the matter distribution. From Gauss' theorem, we conclude that each $\gamma_{\mathrm{ik}}$ is constant ${ }^{13}$ and thus must everywhere adopt the Minkowskian values presumed at spatial infinity.

\title{
3.2 The Attempt to Recover them: The Harmonic Coordinate Condition
}

\author{
Einstein found that the natural choice of gravitation tensor, the Ricci \\ tensor, would yield weak field equations of form (8) if he restricted
}

himself to harmonic coordinates. But the Ricci tensor is rejected since the harmonic coordinate condition is incompatible with the spatial

flatness Einstein presumed for weak, static fields.

Einstein now needed to find a gravitation tensor for his field equations (7) that would revert to (8) in the weak field. Grossmann reported the key mathematical result in his part of the "Entwurf" paper (II §2): one generates "the complete system of differential tensors of the manifold" by covariant algebraic and differential operations on what we now call the Levi-Civita tensor density and the fourth rank Riemann curvature tensor $\mathrm{R}_{\mathrm{iklm}}$, where the indices now range over 1,2,3 and 4. The natural candidate for the gravitation tensor was the Ricci tensor, the unique first contraction

$$
G_{i m}=\sum_{k, l} \gamma_{k l} R_{i k l m}=\sum_{k, l} \gamma_{k l} \frac{1}{2}\left(\frac{\partial^{2} g_{i m}}{\partial x_{k} \partial x_{l}}+\frac{\partial^{2} g_{k l}}{\partial x_{i} \partial x_{m}}-\frac{\partial^{2} g_{i l}}{\partial x_{k} \partial x_{m}}-\frac{\partial^{2} g_{m k}}{\partial x_{l} \partial x_{i}}\right)+\begin{gathered}
\text { terms quadratic } \\
\text { first derivatives } \\
\text { of }
\end{gathered}
$$

\footnotetext{
${ }^{13}$ Pick one component $\gamma_{\mathrm{ik}}$. For a sphere centered on the sun and for a radial coordinate r, Gauss' theorem tells us that $\int_{A} \frac{\partial \gamma_{i k}}{\partial r} d S=\int_{V} \Delta \gamma_{i k} d V=\int_{V} T_{i k} d V=0$ where $\mathrm{A}$ is the area of the sphere and $\mathrm{V}$ its volume. Therefore $\frac{\partial \gamma_{i k}}{\partial r}=0_{\text {so }}$ that, allowing for spherical symmetry, $\gamma_{i k}$ is constant.
} 
This choice is familiar to modern readers since it coincides with the final field equations in the source free case of $\mathrm{T}_{\mathrm{ik}}=0$. But, Grossmann reported, this choice must be abandoned since it fails to yield the Newtonian expression $\Delta \varphi$ in the special case of a weak, static field.

Whether the Ricci tensor can reduce to this form depends solely on the four second derivative terms displayed above; the terms quadratic in the first derivatives can be neglected as second order terms in the weak field approximation. Of these four second derivative terms, the first term alone is sufficient to yield a field equation of the form (8) in the weak field. To assure recovery of (8), the remaining second derivative terms must be eliminated.

What Einstein and Grossmann did not indicate in the "Entwurf" paper was that they knew precisely how this could be achieved. But Einstein's private calculations of the Zurich Notebook do reveal it. If one restricts the spacetime coordinate systems under consideration, these three terms can be made to vanish. In particular, they vanish if one selects the coordinates that satisfy the harmonic condition ${ }^{14}$

$$
\sum_{\alpha, \beta} \gamma_{\alpha \beta}\left\{\begin{array}{c}
\alpha \beta \\
\mu
\end{array}\right\}=0
$$

That the three unwanted second order derivative terms in the Ricci tensor vanish follows from another differentiation of the harmonic condition (10) as Einstein shows on p. $37 / 36$ 19L of the Zurich Notebook. ${ }^{15}$

Nonetheless, Einstein and Grossmann report that the Ricci tensor fails to yield the correct Newtonian limit. What had gone wrong? Again the Zurich Notebook supplies the answer as we watch Einstein grapple unsuccessfully with the weak field in the pages following, pp. 38-42/ 36 19R-21R. While the harmonic coordinate condition did reduce the gravitational field equations to the appropriate Newtonian limit (8), the harmonic condition itself proved objectionable. For Einstein expected the metric to reduce to the spatially flat form (4). A short calculation shows that the harmonic condition (10) is not satisfied in the coordinate system used in (4). Without the harmonic coordinate

\footnotetext{
${ }^{14}$ These coordinate were then called "isothermal" and are now commonly called "harmonic" since the coordinate condition (10) is equivalent to one that has the form of a wave equation $\square x_{\mu}=0$.
} 
condition, Einstein could no longer reduce the Ricci tensor to the appropriate Newtonian form. Since he could find no suitable alternative, the Ricci tensor had to be rejected.

It is instructive to see how Einstein's final theory of 1915 avoids inferring to the spatial flatness of a weak static field. Following Einstein (1992, pp.86-89), we set $\mathrm{G}_{\mu \nu}$ equal to the Ricci tensor. Einstein's final field equations of 1915 then do not have the form (7) but are

$$
\mathrm{G}_{\mu \nu}=\mathrm{k}\left(\mathrm{T}_{\mu \nu}-(1 / 2) \mathrm{g}_{\mu \nu} \mathrm{T}\right)
$$

First we restrict the equation to harmonic coordinates and then proceed as above for the case of a source of pressureless, motionless dust. In place of (8a) and (8b) we recover

$$
\Delta \mathrm{g}_{44}=(-\mathrm{k}) \rho_{0} \quad \Delta \mathrm{g}_{11}=\Delta \mathrm{g}_{22}=\Delta \mathrm{g}_{33}=-(-\mathrm{k}) \rho_{0} \quad \Delta \mathrm{g}_{\mu \nu}=0 \text { all other } \mu, \nu
$$

We see immediately from the second set of equations that the components $\mathrm{g}_{11}, \mathrm{~g}_{22}$ and $\mathrm{g}_{33}$ will not in general be constant if $\rho_{0}$ is anywhere non-vanishing so Einstein's earlier inference to spatial flatness is blocked.

15 "p.37" refers to the pagination of the Zurich notebook introduced in Einstein Papers, Vol. 4. "3 6 19L" uses the system of designation associated with the control numbers in the Einstein Archive. It refers to the left hand side of page 19 of the document 3-6, which is the Zurich Notebook. 


\title{
4. The Puzzle of the Second Candidate
}

\author{
In the Zurich Notebook, Einstein found a second gravitation tensor of \\ broad covariance that yielded the appropriate Newtonian limit and the \\ spatial flatness of weak, static fields. It was briefly revived in \\ November 1915. What explains its rejection in 1913 and revival in
}

1915?

Einstein's presumption of the spatial flatness of weak, static fields was sufficient to preclude his

consideration of the Ricci tensor. But it does not explain why he ended up abandoning general covariance in 1913.

The field equations he announced in the "Entwurf" paper were of unknown covariance and Einstein could assert at best a near trivial covariance under linear coordinate transformations. In this regard, the Zurich Notebook contains a puzzle. Immediately after the harmonic condition was abandoned, on p. 44/ $3622 \mathrm{R}$ Einstein found a reduced form of the Ricci tensor with very broad covariance that could be used as a gravitation tensor and, with a suitable choice of coordinate condition, would yield the equation (8) in the weak field. In this instance, the coordinate condition was compatible with the spatially flat metric (4), so none of the difficulties we have seen so far preclude acceptance of it as the gravitation tensor. That tensor proved so unobjectionable that Einstein later came to endorse it briefly in publication. When he returned to general covariance in late 1915, but before he realized his error concerning the spatial flatness of weak, static fields, Einstein (1915) published field equations using this very gravitation tensor.

The puzzle is this: why were these equations inadmissible in 1913 but admissible briefly ${ }^{16}$ in November 1915. Some additional error must explain it. What was it?

\footnotetext{
${ }^{16}$ That Einstein's public endorsement of them in 1915 was brief is readily explained by his recognition over the weeks following that weak, static fields need not be spatially flat, so that the Einstein tensor became admissible as a gravitation tensor and was quickly chosen by him.
} 


\subsection{Construction of the New Candidate Gravitation Tensor}

Einstein splits off a gravitation tensor of near general covariance from

the Ricci tensor and shows how to reduce it to the required Newtonian

form by application of a coordinate condition.

While the details of the construction of this gravitation tensor are inessential for the conjecture to follow, it is included here briefly for completeness. Einstein noted that the Ricci tensor could be written as a sum of two $\operatorname{parts}^{17}$

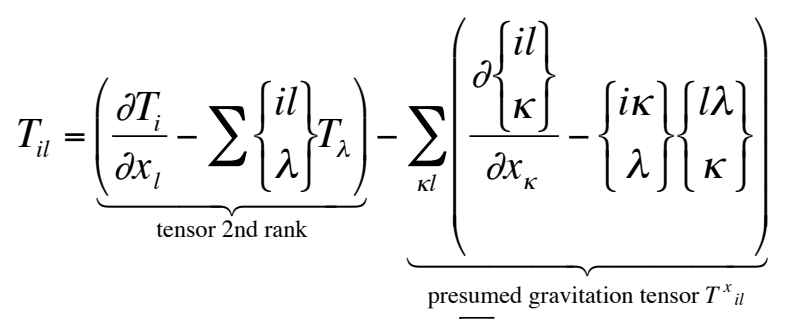

where the quantity $\mathrm{T}_{\mathrm{i}}$ of the first term is defined as $T_{i}=\frac{\partial \lg \sqrt{G}}{\partial x_{i}}$ with $\mathrm{G}$ the determinant of the metric tensor. A unimodular transformation of the spacetime coordinates $\mathrm{x}_{\alpha} \rightarrow \mathrm{x}_{\beta}^{\prime} \beta$ is one for which the determinant $\operatorname{Det}\left(\frac{\partial x_{\beta}^{\prime}}{\partial x_{\alpha}}\right)=1$.

It follows that unimodular transformations preserve $\mathrm{G}$ which becomes a scalar. Immediately we have that $\mathrm{T}_{\mathrm{i}}$ is a vector under unimodular transformation since it is just the derivative of a scalar. The first quantity in the expression for the Ricci tensor $\mathrm{T}_{\mathrm{il}}$ proves to be the covariant derivative of this vector and thus also a tensor of second rank under unimodular transformation. Since $\mathrm{T}_{\mathrm{il}}$ is a generally covariant tensor, it now follows that the second term must also be a tensor under unimodular transformation. Labeling the second term $\mathrm{T}^{\mathrm{x}}{ }_{\mathrm{i}}$, Einstein adopted it as the gravitation tensor.

This tensor is not generally covariant, but its covariance is sufficiently broad to support Einstein's ambitions for generalizing the principle of relativity to acceleration. Unimodular transformations include those that set the Cartesian spatial coordinate axes of a Minkowski spacetime into uniform rotation, for example (transformation (12) below).

${ }^{17}$ The form given is quoted directly from the Zurich notebook and the annotations on the terms are Einstein's. 
Like the Ricci tensor, the new candidate gravitation tensor contained more second derivative terms in the metric tensor than present in the weak field equation (8). As before Einstein eliminated them with a coordinate condition. This time he chose the simple condition

$$
\sum_{\kappa} \frac{\partial \gamma_{\kappa \alpha}}{\partial x_{\kappa}}=0
$$

and was able to show that in coordinate systems in which it holds, a gravitational field equation based on $\mathrm{T}^{\mathrm{x}}{ }_{\mathrm{il}}$ reduces to the desired form (8). Finally one can see by inspection that the coordinate condition (11) is satisfied in the weak static field (4).

\title{
4.2 Einstein's "Fateful Prejudice"
}

\author{
Part of Einstein's rejection of this second candidate was due to his \\ "fateful prejudice" concerning the Christoffel symbols. I discount the \\ possibility that the rejection can be explained by the supposition that he \\ was unaware of the standard use of coordinate conditions.
}

Many factors may have entered into Einstein's decision to abandon this second candidate. In this paper I will discuss just one possibility of special interest. There are others. In his later remarks of November 1915, Einstein blamed the decision on a "fateful prejudice." Its most expansive description come in a letter to Sommerfeld of November 28, 1915 (Papers, Vol. 8A, Doc. 153.). There he reflected ruefully on field equations that used the second candidate tensor: ${ }^{18}$

I had already considered these equations 3 years ago with Grossmann... but had then arrived at the result that they did not yield Newton's approximation, which was erroneous. What supplied the key to this solution was the realization that it is not

$$
\sum g^{l \alpha} \frac{\partial g_{\alpha i}}{\partial x_{m}}
$$

but the associated Christoffel symbols $\left\{\begin{array}{c}\mathrm{im} \\ 1\end{array}\right\}$ that are to be looked upon as the natural expression for the "components" of the gravitational field. If one sees this,

\footnotetext{
18 There are similar remarks in the paper Einstein (1915, p.1056)
} 
then the above equation becomes simplest conceivable, since one is not tempted to transform it by multiplying out the symbols for the sake of general interpretation.

Our best interpretation of this depends upon insights by Jürgen Renn (forthcoming). They pertain to a difficulty in assuring energy momentum conservation in a theory based on this gravitation tensor. For it to be assured, Einstein required that he be able to define a stress-energy tensor for the gravitational field. The prejudice Einstein outlined induced him to seek an expression for it in terms of the derivatives of the metric tensor. That yielded a calculation so daunting that Einstein abandoned it. By 1915, after he had developed more powerful variational techniques, Einstein found that this quantity could be expressed more simply in terms of the Christoffel symbols and the difficulty disappeared. For further discussion, see Norton (forthcoming).

It is quite improbable that this was the only difficulty faced by this second candidate gravitation tensor. Otherwise we must assume that Einstein gave up at his moment of triumph simply because the calculation look hard. Also we would have no explanation for his remark to Sommerfeld above that the equations did not yield the Newtonian limit. There must have been a further problem of sufficient gravity to thwart Einstein completely.

The pages surrounding the analysis of this second candidate gravitation tensor in the Zurich notebook are concerned with problems of coordinates and covariance. There it becomes clear that Einstein is not using coordinate condition (11) and others like it in the now standard way. He was not merely invoking the condition in the special case of the Newtonian limit. (For that usage, we reserve the label "coordinate condition.") Rather he was invoking it universally, so that the resulting reduced form of the gravitational field equations were not just to be used in the weak field limit. They were the theory's gravitational field equations. To distinguish this usage from the standard use, we have come to call equations such as (11) used this way "coordinate restrictions." This interpretation of Einstein's use of (11) and the label “coordinate restriction” was foreshadowed in in Renn and Sauer (1999, p. 108) and elaborated in Renn, Sauer et al. (forthcoming).

That Einstein sometimes used the requirement (11) as a coordinate restriction does not explain why he might think that the second candidate gravitation tensor fails to yield the Newtonian limit. A stronger supposition is needed. We must presume in addition that Einstein was unaware of the other way of using the requirement as a coordinate condition. A case can be made that this awareness defeated recovery of the Newtonian limit. For if Einstein tried to use requirement (11) as a coordinate restriction in the attempt to recover the Newtonian limit, the 
covariance of the final field equations would be reduced to that of requirement (11). We shall see below in Section 6.1 that requirement (11) has insufficient covariance to support an extension of the principle of relativity. However I do not find this supposed lack of awareness plausible for reasons given in some detail in Norton (forthcoming). ${ }^{19}$ Briefly, it requires Einstein to fail persistently to see that he may impose a restriction on covariance in setting up the special conditions needed for recovery of the Newtonian limit, just as he may impose the assumption of near Minkowskian values for the metric tensor. He must overlook this in spite his continued insistence that the restricted of covariance Newtonian theory is what distinguishes it fundamentally from his new theory and that covariance principles are his area of greatest insight and expertise. Also Einstein makes no later concession of an error of this type and is very careless in his introduction of coordinate conditions to the point of obscuring their presence, an attitude that is odd if their neglect proved fatal to his earlier efforts.

The alternative conjecture to be developed in the sections following draws on the same base of evidence and does require Einstein to commit an error concerning coordinate systems and coordinate conditions. But the error attributed to Einstein is one that we see him committing unequivocally later and to which he also later admits. The conjecture just requires that he committed the same error earlier and pursued its consequences.

19 The supposed unawareness is incompatible with Einstein's labeling of terms on p. 44/ $3622 \mathrm{R}$. He introduces the decomposition of the Ricci tensor apparently aware in advance that one part, the quantity $\mathrm{T}^{\mathrm{x}}{ }_{\mathrm{il}}$, will reduce to the Newtonian form (8) under imposition of the requirement (11). If (11) is not being used as a coordinate condition, his gravitation tensor is whatever $\mathrm{T}^{\mathrm{x}}{ }_{\mathrm{il}}$ reduces to after imposition of (11). Yet Einstein carefully and clearly labels $\mathrm{T}^{\mathrm{x}}{ }_{\mathrm{il}}$ as "presumed gravitation tensor" - just the appropriate labeling if (11) is being used as a coordinate condition. 


\section{The Hole Argument}

Einstein's other error from this period was his "hole argument," which appeared months later. With it he sought to establish that generally covariant gravitational field equations would be physically

uninteresting.

To understand why the gravitation tensor $\mathrm{T}^{\mathrm{x}}{ }_{\mathrm{il}}$ was inadmissible in 1913 but not in early November 1915 , we must locate some new error on Einstein's part--that is, an assumption that Einstein himself would later regard as erroneous. Rather than needing to locate a new, hitherto unknown error, my conjecture is that the error Einstein later conceded in the context of his notorious hole argument can also explain Einstein's earlier rejection of the gravitation tensor $\mathrm{T}_{\mathrm{il}}$. At the same time, it will reveal just how difficult Einstein had made his search for any admissible, generally covariant gravitation tensor and that the search's failure in 1913 was all but assured until that error was corrected.

Once Einstein had published gravitational field equations of very limited covariance in 1913, he needed to convince his readers and correspondents that this choice was acceptable. After some vacillation, ${ }^{20}$ he settled upon the hole argument for this task. While the "Entwurf" paper was published in mid 1913, Einstein does not seem to have had the hole argument in hand until months later. The first unambiguously dated mention of it is in a letter to November 2, 1913, to Ludwig Hopf (Papers, Vol. 5, Doc. 480). In the ensuing year, Einstein published the argument four times, with the final version in Einstein (1914, p. 1067) being the clearest.

\footnotetext{
${ }^{20}$ For an account of the vacillations see Norton $(1984, \S 5)$. For further discussion see also Stachel (1980, §§3-4), Norton (1987).
} 


\subsection{Outline of the Argument}

A generally covariant gravitation theory is inadmissible since a full specification of the metric field outside some small region (the "hole")

cannot fix the metric field within it.

The purpose of the argument was to show that a version of Einstein's theory with generally covariant gravitational field equations would violate what he called the "law of causality" (Einstein, 1914, p.1066). In effect he meant that the theory would be indeterministic. That is, a full specification of the metric field outside some region of spacetime must fail to fix the metric field within that region, no matter how small the region may be.

In slightly simplified form, Einstein's argument proceeded as follows. ${ }^{21}$ Let us assume that the metric field in the source free case is governed by generally covariant gravitational field equations $\mathrm{G}_{\mu \nu}=0$ and that we have a solution of these equations $g_{\mu \nu}$ in some coordinate system $x_{\alpha}$. Since the field equations $G_{\mu \nu}=0$ are generally covariant, any arbitrary transform of $g_{\mu \nu}$ will also be a solution of these field equations. Consider the following transformation. We select some arbitrary region of spacetime--call it the "hole." The transformation is the identity outside the hole, but comes smoothly to differ from the identity within the hole; it maps a point $\mathrm{P}$ with coordinates $\mathrm{x}_{\alpha}$ to a point $\mathrm{Q}$ with coordinates $\mathrm{x}_{\beta}^{\prime}=\mathrm{f}_{\beta}\left(\mathrm{x}_{\alpha}\right)$ in the same coordinate system. Outside the hole $\mathrm{P}=\mathrm{Q}$; inside $\mathrm{P} \neq \mathrm{Q}$. We apply this active transformation to the metric $g_{\mu \nu}$ and thereby generate another solution of the gravitational field equation $g^{\prime} \mu \nu$ in the same coordinate system $x_{\alpha}$. The transformation is displayed in Figure 5 in which the metric field is represented by the light cones and timelike geodesics it induces on the spacetime.

\footnotetext{
${ }^{21}$ The simplification is that I consider a matter free metrical field, whereas Einstein considered a source matter distribution in which the hole is a matter free region.
} 


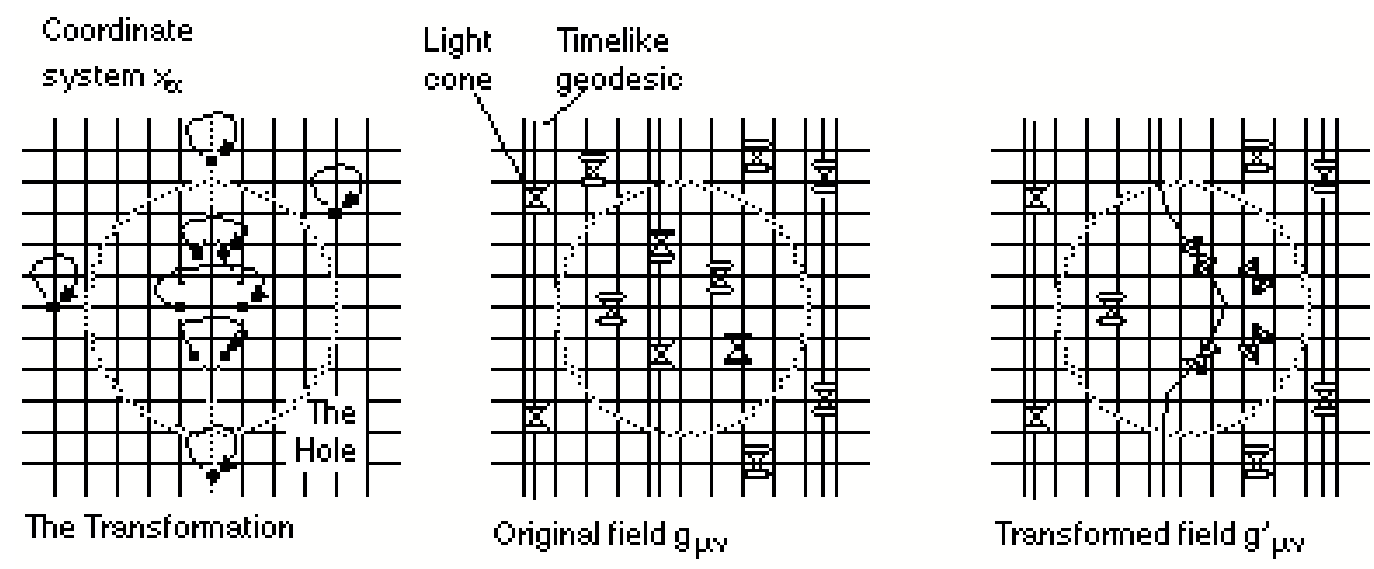

Figure 5. Transforming Fields for the Hole Argument

To arrive at the violation of determinism, we begin with the solution $g_{\mu v}\left(x_{\alpha}\right)$ in the coordinate system $x_{\alpha}$. We imagine this field removed from the coordinate system and then replaced by the transformed field $g^{\prime} \mu v\left(x_{\alpha}\right)$ as shown in Figure 6.
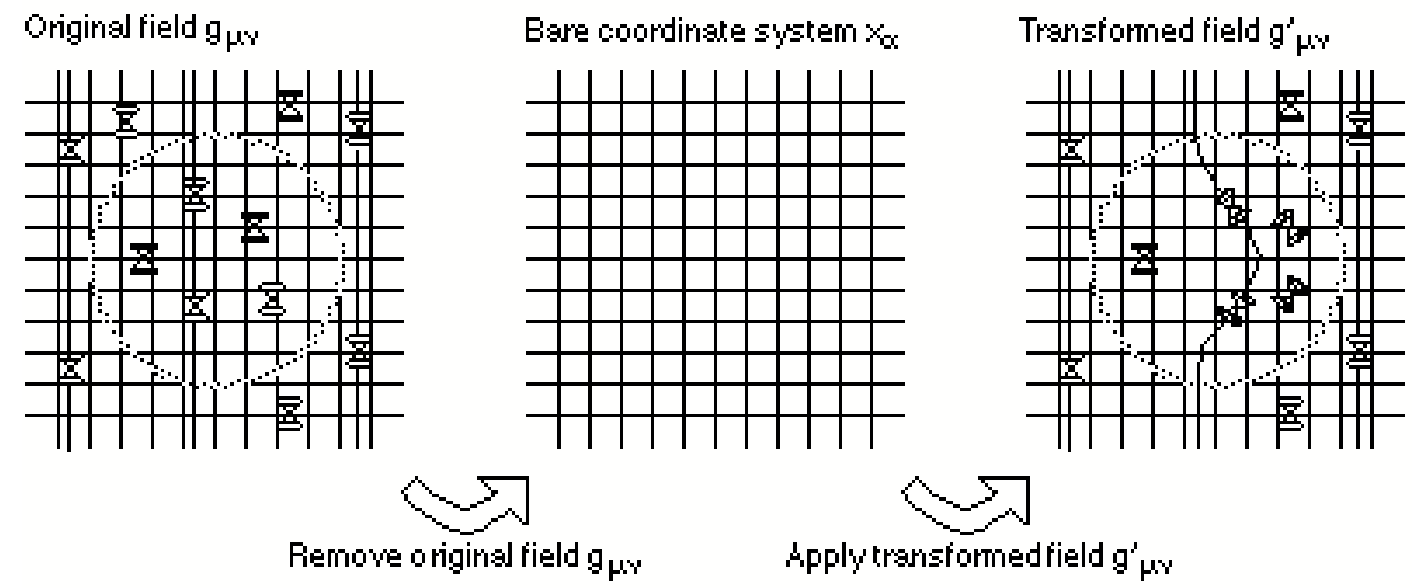

Figure 6. The Manipulation of the Hole Argument

If we compare the two solutions, we find they agree fully outside the hole since the transformation is the identity there, but they disagree within. That is, if we specify the metric fully outside the hole, we cannot know which field we will find within. This is a failure of determinism so severe that Einstein felt it must be suppressed. That, he urged, was achieved by disavowing the general covariance of the gravitational field equations. 


\subsection{Active versus Passive Transformations}

It proved easy to overlook that Einstein intended the transformation of the hole argument to be read actively so that it left the coordinate system unchanged but spread the metric differently over it.

Einstein's earlier discussion of this construction caused considerable confusion among later commentators and was only made completely explicit in the version of Einstein (1914, p.1067). To generate the active transformation, Einstein first read the transformation passively as a change of coordinate system and it proved easy to overlook the crucial conversion of that passive transformation into an active transformation of the field in just one coordinate system.

He began by using the transformation represented by the functions $\mathrm{f}_{\beta}$ as a passive transformation to relabel the point $\mathrm{P}$ by with new coordinates $\mathrm{x}_{\beta}^{\prime}$. Under this coordinate transformation, the components of the tensor $\mathrm{g}_{\mu \nu}\left(\mathrm{x}_{\alpha}\right)$ transform to components $g_{\mu \nu}^{\prime}\left(x_{\beta}^{\prime}\right)$ in the new coordinate system $x_{\beta}^{\prime}$ following the usual law of transformation of tensor components. To proceed to the active transformation, Einstein considered the functional dependence of the transformed $g_{\mu v}^{\prime}\left(x_{\beta}^{\prime}\right)$ on its arguments, the coordinates $x^{\prime} \beta$. That functional dependence alone was all that was needed to assure that the field $g^{\prime} \mu \nu$ satisfies the field equations $G_{\mu \nu}=0$. Those same functional forms realized in any other coordinate system would then also represent a solution of the field equations. Thus the new field $g^{\prime} \mu v\left(x_{\alpha}\right)--$

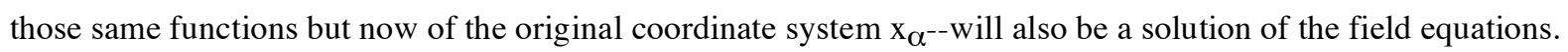
This new field is the active transform $g_{\mu \nu}^{\prime}\left(x_{\alpha}\right)$ of the original field $g_{\mu \nu}\left(x_{\alpha}\right)$ with both represented in the original coordinate system $x_{\alpha}$.

As a trivial illustration of the conversion to the active transformation, imagine that the functions $g^{\prime} \mu v\left(x^{\prime} \beta\right)$ just happen to be the constant functions of the arguments $x_{\beta}^{\prime}$. Then we know that constant $g_{\mu \nu}^{\prime}=K_{\mu \nu}$ solve the field equations $\mathrm{G}_{\mu \nu}=0$ and that will be true no matter which coordinate system we consider. So, take the original coordinate system $x_{\alpha}$ and construct a new field in it whose components $g^{\prime} \mu v\left(x_{\alpha}\right)=K_{\mu \nu}$ are those same constants. The new field will be distinct from the original field $g_{\mu v}\left(x_{\alpha}\right)$ but will still be a solution of the gravitational field equations.

For another development of the mathematical constructions used in the hole argument, see Howard and Norton $(1993, \S 1)$. 


\title{
5.3 The Erroneous Assumption: Independent Reality of Spacetime Coordinate Systems
}

\author{
Einstein error, as he later explained, was that he believed that the \\ spacetime coordinate system had an existence independent of the \\ metrical field defined on it so that it made sense for the same \\ coordinate system to host distinct metric field.
}

Or course Einstein's use of the hole argument is flawed. It does not force us to abandon general covariance. An easier escape simply allows that the two fields $g_{\mu \nu}$ and the active transform $g^{\prime} \mu \nu$ are distinct mathematical representations of the same physical reality. Therefore the hole argument fails to show that the physically real within the hole is underdetermined; it merely shows failure of determinism for the mathematical structures we choose to s failure of determinism for the mathematical structures we choose to describe the one physical reality.

Why do $g_{\mu \nu}$ and $g_{\mu \nu}^{\prime}$ represent the same physical reality? Since they are transforms of one another they must agree on all invariants. So if elements of physical reality are represented only by invariants, the two field represent the same physical reality. Einstein's preferred formulation of this escape is to note that two intertransformable systems agree on all point coincidences. For example, if the world consisted just of particles in motion, the intersections of their worldlines, he asserted, would be the only observable and they would be preserved under all transformations. This is Einstein's "point-coincidence argument," best know from his review article, Einstein $(1916, \S 3)$.

For our purposes, however, what is most important is not the correct analysis of the hole argument but the error Einstein committed that prevented him seeing the correct analysis. That error was explained by Einstein to his correspondents late in 1915 and in early 1916. The difficulty pertains to the coordinate system that carries the fields. For example in a letter to his friend Michele Besso a little over a week later on January 3, 1916 (Papers, Vol. 8A, Doc. 178; Einstein's emphasis) he explained:22

\footnotetext{
${ }^{22}$ Einstein's formulae $\mathrm{G}(\mathrm{x})$ and $\mathrm{G}^{\prime}(\mathrm{x})$ correspond to $\mathrm{g}_{\mu \nu}\left(\mathrm{x}_{\alpha}\right)$ and $\mathrm{g}_{\mu \nu}^{\prime}\left(\mathrm{x}_{\alpha}\right)$ respectively. Einstein sent a very similar explanation to Paul Ehrenfest in a letter of December 26, 1915 (Papers, Vol.8a, Doc. 173).
} 
There is no physical content in two different solutions $\mathrm{G}(\mathrm{x})$ and $\mathrm{G}^{\prime}(\mathrm{x})$ existing with respect to the same coordinate system K. To imagine two solutions simultaneously in the same manifold has no meaning and the system $\mathrm{K}$ has no physical reality.

The error Einstein identifies concern what happens at some particular quadruple of values $\mathrm{x}_{\alpha}$ in the coordinate system. The naive reading is that this quadruple picks out a particular physical event in spacetime and that the two solutions $g_{\mu v}\left(x_{\alpha}\right)$ and $g_{\mu v}^{\prime}\left(x_{\alpha}\right)$ attribute different metrical properties to that event. This naive reading is mistaken. The quadruple $\mathrm{x}_{\alpha}$ does not pick out any particular physical event until a metrical field is defined on the coordinate system. Only then can it do so. As a result the two solutions $g_{\mu v}\left(x_{\alpha}\right)$ and $g_{\mu v}^{\prime}\left(x_{\alpha}\right)$ do not necessarily ascribe different metric properties to the same physical event. Thus a coordinate system is something less than we may naively think. It coordinates with nothing until a metric is defined on it. That is, take the metric off and one is not left with a coordinate system that labels the physical events of reality; that labeling is gone and the coordinate system as a labeling device ceases to function. In Einstein's words the "[coordinate] system... has no physical reality." We might phrase this more cautiously by saying that the coordinate system has no reality independent of the metric, for the combination of coordinate system and metric certainly do represent aspects of physical reality. In terms of the construction of the hole argument represented in Figure 6, the error is to think that the bare coordinate system $\mathrm{x}_{\alpha}$ remains and continues to label the same physical events once the metric $\mathrm{g}_{\mu \nu}$ is removed and that is can then host the new field $g_{\mu \nu}^{\prime}$. What really would happen if we could somehow remove the metric field $g_{\mu \nu}$ from the coordinate system is shown figuratively in Figure 7. 


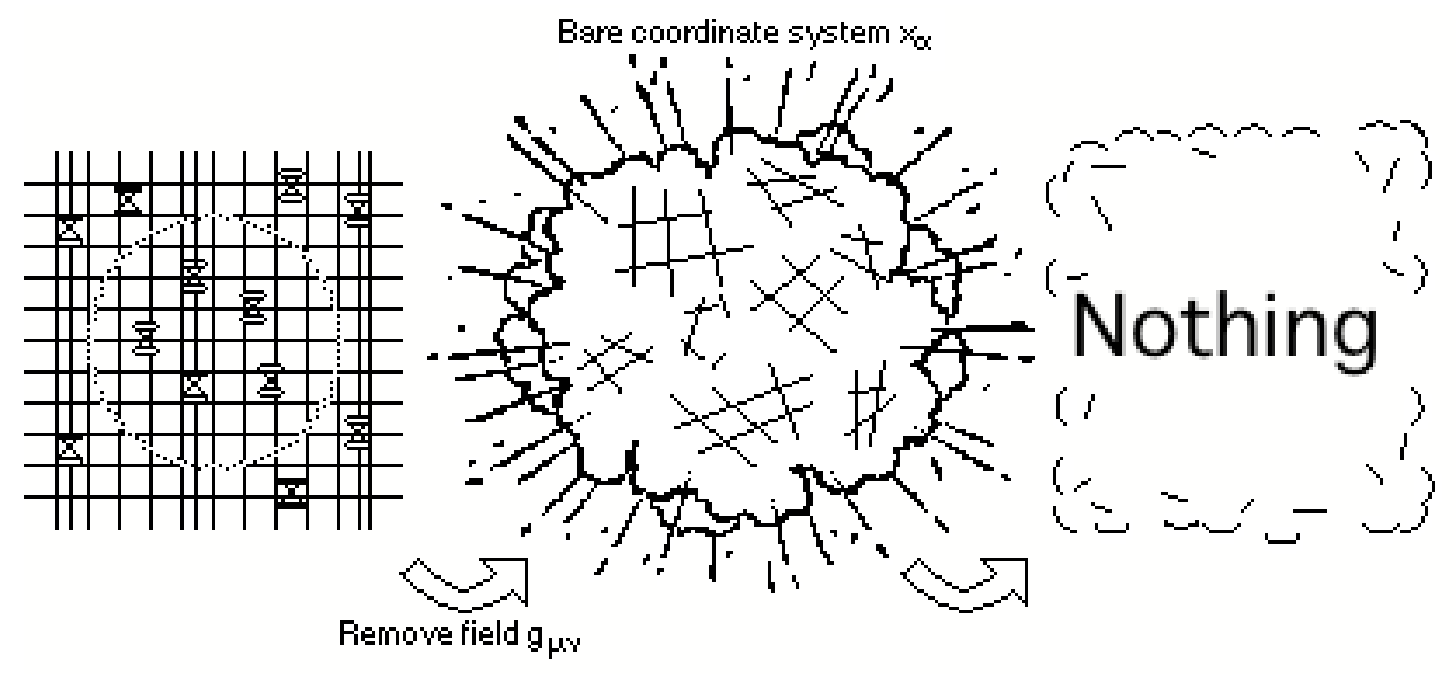

Figure 7. Failure of the Hole Argument

This rather melodramatic portrayal may well not be so far from the way that Einstein himself visualized his error. Years later, after the sharply positivistic tone in his writing had much blunted, he wrote even more sharply about what happens if we imagine the removal of the metric field. A 1952 appendix "Relativity and the Problem of Space" to his popular text Relativity gives his mature view of the issues addressed hastily to his correspondents in late 1915 and early 1916. He wrote (Einstein, 1954, p.155; Einstein's emphasis):

On the basis of the general theory of relativity, on the other hand, space as opposed to "what fills space", which is dependent on the co-ordinates, has no separate existence. Thus a pure gravitational field might have been described in terms of the $g_{i k}$ (as functions of the coordinates), by solution of the gravitational equations. If we imagine the gravitational field, i.e. the functions $g_{i k}$, to be removed, there does not remain a space of the type (1) [23], but absolutely nothing and also no topological space.

Finally it is important to note the tacit character of Einstein's error. He could not have been consciously aware that his hole argument depended essentially on according an independent reality to the coordinate systems. If

\footnotetext{
${ }^{23}$ Einstein's formula (1) is the line element $\mathrm{ds}^{2}=\mathrm{dx}_{1}{ }^{2}+\mathrm{dx}_{1}{ }^{2}+\mathrm{dx}_{1}{ }^{2}-\mathrm{dx}_{4}{ }^{2}$ of special relativity. In continuing to explain how general relativity regards a space with this line element, he repeats what for present purposes is the key insight learned in Einstein's 1915 rejection of the hole argument: "...the coordinate system used ... in itself has no objective significance..." (p.155).
} 
he had noticed it, he would surely have rejected the supposition. Einstein's explanations of the transformations in the early expositions of the hole argument are sufficiently hasty to obscure their true character. This reflects his inattention to the presumptions on which they depend. 


\title{
6. The Conjecture
}

\author{
If Einstein erroneously accorded the same independent reality to the \\ restricted class of coordinate systems in which the Newtonian limit is \\ realized, then these coordinate systems would adopt the same
}

objectionable absolute properties as the preferred inertial coordinate

systems of special relativity, rendering the candidate gravitational field

equations inadmissible.

Why did Einstein so rapidly forsake the gravitation tensor $\mathrm{T}^{\mathrm{x}}{ }_{\mathrm{il}}$ during his preparation of the "Entwurf"

paper? Why did he recall to Sommerfeld that he thought it would not return the Newtonian limit, when the calculation of p.44/ $3622 \mathrm{R}$ shows just how this can be done? We should expect to find clues on the pages of the Zurich notebook surrounding p.44/3 $622 \mathrm{R}$ where the gravitation tensor $\mathrm{T}^{\mathrm{x}}{ }_{\mathrm{il}}$ appears. These pages deal with coordinate conditions and their transformation properties. On the pages following p.44/3 $622 \mathrm{R}$, one particular transformation is given special attention, the transformation that sets the Cartesian spatial coordinate axes $(\mathrm{x}, \mathrm{y}, \mathrm{z})$ of the Minkowski spacetime (2) into uniform rotation

$$
x^{\prime}=x \cos \omega t+y \sin \omega t \quad y^{\prime}=-x \sin \omega t+y \cos \omega t \quad z^{\prime}=z \quad t^{\prime}=t
$$

While that is unremarkable, something more incongruous is on p. $43 / 3622 \mathrm{~L}$, the page facing p. $43 / 3622 \mathrm{R}$. There Einstein investigates the covariance properties of the requirement (11) under non-linear, unimodular transformations. (The rotation transformation (12) is a non-linear, unimodular transformation.) That would not be surprising if Einstein was merely using the requirement as a coordinate restriction. But might it also be revealing some defect perceived by Einstein in requirement (11) if it is to be used as a coordinate condition?

If the coordinate condition (11) is used in the modern way, there would be no point in an investigation of its covariance The coordinate condition is merely used to reduce the gravitational field equations to their Newtonian form in some restricted set of coordinate systems-let us call them ${ }_{x}{ }^{\operatorname{LIM}}{ }_{\alpha}$. The condition need not have any more covariance than the Galilean covariance of Poisson's equation (6). One sees with minimal calculation that the condition (11) is not merely covariant under Galilean transformation but under any linear transformation of the 
coordinates. ${ }^{24}$ Might Einstein's concern with the covariance properties of this coordinate condition reveal why he mistakenly thought its use in recovering the Newtonian limit a failure?

\title{
6.1 The Independent Reality of the Spacetime Coordinate System of the Newtonian Limit
}

\author{
Using the same construction as in Figure 6, Einstein would find that \\ the limiting coordinate systems $x^{L I M}{ }_{\alpha}$ admits the special relativistic \\ field $\eta_{\mu v}(2)$ but not the rotation field $g^{R O T} \mu \nu(13)$ because of the \\ insufficient covariance of the coordinate condition (11).
}

My conjecture is that, in 1913, Einstein may have harbored a different understanding of the coordinate condition (11) and the coordinate systems $\mathrm{x}^{\mathrm{LIM}}{ }_{\alpha}$ that they pick out. That difference is just the error Einstein later conceded in the context of the hole argument. That is, Einstein treated the coordinate systems $\mathrm{x}^{\mathrm{LIM}}{ }_{\alpha}$ as physically real elements within his theory, whose existence is independent of the metric fields defined on them. In particular, this means that it is possible to reproduce with them exactly the construction depicted in Figures 5 and 6 . He could consider one solution of the gravitational field equations in a coordinate system $x^{\mathrm{LIM}}{ }_{\alpha}$, imagine that field removed and then a new, transformed field applied to the very same coordinate system.

Let us consider this construction in the simple case suggested by Einstein's concern for the rotation transformation (12). We begin with the Minkowski metric $\eta_{\mu \nu}$ shown as (2), which is the metric Einstein associated with special relativity. ${ }^{25} \mathrm{We}$ (actively) transform it under the rotation transformation (12). The result is the rotation field $\mathrm{g}^{\mathrm{ROT}}{ }_{\mu \nu}$ whose components are

${ }^{24}$ Under a linear transformation from coordinate system $\mathrm{x}_{\alpha}$ to $\mathrm{x}_{\beta}^{\prime}$, the coefficients $p_{\alpha}{ }^{\beta}=\frac{\partial x_{\beta}^{\prime}}{\partial x_{\alpha}}$ and $\pi_{\beta}{ }^{\alpha}=\frac{\partial x_{\alpha}}{\partial x^{\prime}}$ are constants and this constancy is all that is needed to secure the covariance of (11). If condition(11) holds in unprimed coordinates $\sum_{\kappa} \frac{\partial \gamma_{\kappa \alpha}}{\partial x_{\kappa}}=0$ then so does condition (11) in primed coordinates since $\sum_{\mu} \frac{\partial \gamma_{\mu \beta}^{\prime}}{\partial x_{\mu}^{\prime}}=\sum_{\mu \alpha} \pi_{\mu}{ }^{\lambda} \frac{\partial}{\partial x_{\lambda}}\left(p_{\kappa}{ }^{\mu} p_{\alpha}{ }^{\beta} \gamma_{\kappa \alpha}\right)=\sum_{\mu \alpha} \pi_{\mu}{ }^{\lambda} p_{\kappa}{ }^{\mu} p_{\alpha}{ }^{\beta} \frac{\partial \gamma_{\kappa \alpha}}{\partial x_{\lambda}}=\sum_{\alpha} p_{\alpha}{ }^{\beta} \sum_{\kappa} \frac{\partial \gamma_{\kappa \alpha}}{\partial x_{\kappa}}=0$

${ }^{25}$ While this is no longer the practice in relativity theory, Einstein then considered special relativity not just as the case of a Minkowski spacetime, but as a Minkowski spacetime in the inertial coordinate system associated with (2). 


$$
g_{\mu \nu}^{R O T}=\left[\begin{array}{cccc}
-1 & 0 & 0 & \omega y \\
0 & -1 & 0 & -\omega x \\
0 & 0 & -1 & 0 \\
\omega y & -\omega x & 0 & 1-\omega^{2}\left(x^{2}+y^{2}\right)
\end{array}\right]
$$

The transformation is shown in Figure 8, where the metric field is represented as before by the light cones and timelike geodesics it induces on the spacetime. The field $\mathrm{g}^{\mathrm{ROT}}{ }_{\mu v}$ is a rotation field in the sense that free particles follow helical worldlines in the coordinate system $\mathrm{x}_{\alpha}$ that rotate around a central axis.

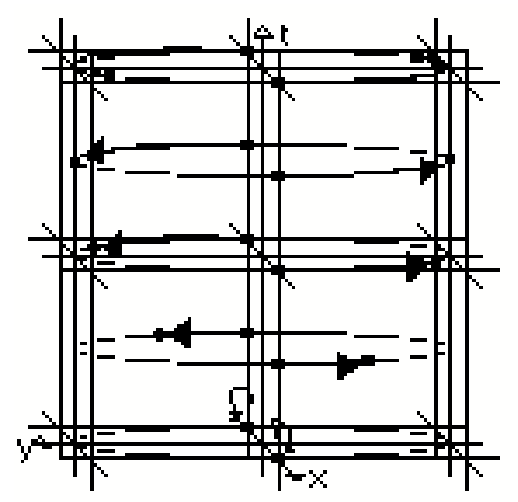

Rotation transformation

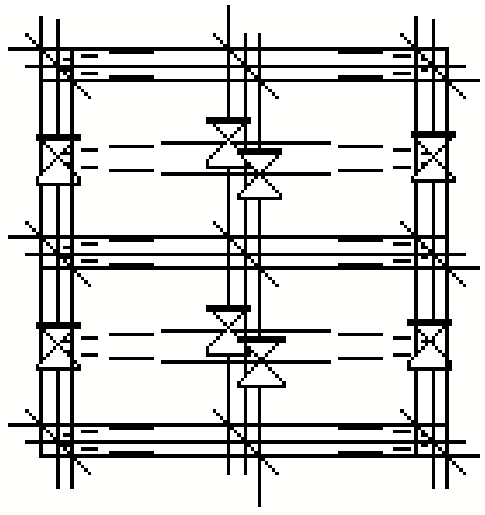

Original metric field $n_{\mu v}$

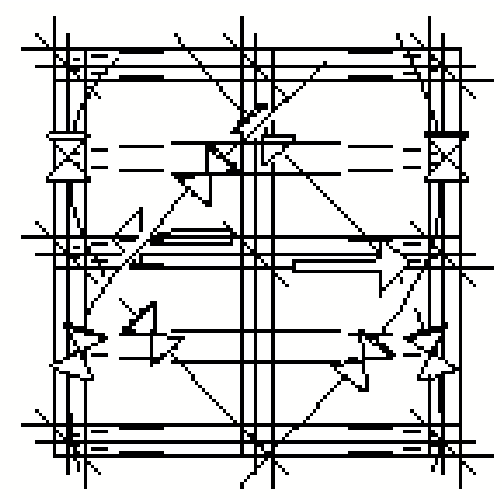

Transformed metric field $g \mathrm{ROT}{ }_{\mu \mathrm{v}}$

Figure 8. Rotation transformation (12) creates a rotation field

The metric $\eta_{\mu \nu}$ has constant components. So we know without calculation that it is admissible in the coordinate system $x^{\text {LIM }} \alpha^{--i t}$ satisfies both the source free field equations $T^{x_{i l}=0}$ and the condition that picks out $x^{\text {LIM }}{ }_{\alpha}$, the coordinate condition (11). We remove the metric field $\eta_{\mu \nu}$ and seek to apply the rotation field gROT $\mathrm{R}_{\mu \nu}$ to the bare coordinate system $x^{\text {LIM }}{ }_{\alpha}$ as shown in Figure 9. Will ${ }^{\text {LIM }}{ }_{\alpha}$ admit the rotation field, $g^{\text {ROT }}{ }_{\mu \nu}$ ? Since the field equations $\mathrm{T}^{\mathrm{X}}{ }_{\mathrm{il}}=0$ are covariant under transformation (12), the rotation field $\mathrm{g}^{\mathrm{ROT}}{ }_{\mu v}$ satisfies it. But to be admissible in $\mathrm{x}^{\mathrm{LIM}}{ }_{\alpha}, \mathrm{g}^{\mathrm{ROT}}{ }_{\mu \nu}$ must also satisfy the coordinate condition (11). A short calculation shows that it does not. We find

The rotation field is by modern lights just another presentation of Minkowski spacetime, but Einstein treated it as a different case. This is not the place to debate whether this approach is viable. Our concern is to understand Einstein's reasoning at that time. For discussion of the rationale underlying Einstein's approach see Norton (1989), (1992a) and (1993). 


$$
\sum_{\kappa} \frac{\partial \gamma_{\kappa}^{R O T} \kappa \alpha}{\partial x_{\kappa}}=\left(-\omega^{2} x,-\omega^{2} y, 0,0\right) \neq 0
$$

so that (11) fails and the rotation field $\mathrm{g}^{\mathrm{ROT}}{ }_{\mu \nu}$ in inadmissible in the coordinate system $\mathrm{x}^{\mathrm{LIM}}{ }_{\alpha}$. This is the most direct way to arrive at the result. There is another indirect path. The coordinate condition (11) is satisfied by the metric $\eta_{\mu v}$. It will also be satisfied by the rotation field $\mathrm{g}^{\mathrm{ROT}}{ }_{\mu \nu}$ if coordinate condition (11) is covariant under the rotation transformation (12). This transformation is non-linear and unimodular. So an alternate calculation is to test the covariance of coordinate condition (11) under non-linear, unimodular transformation, just as Einstein does on the facing page p. $43 / 3622 \mathrm{~L}$.

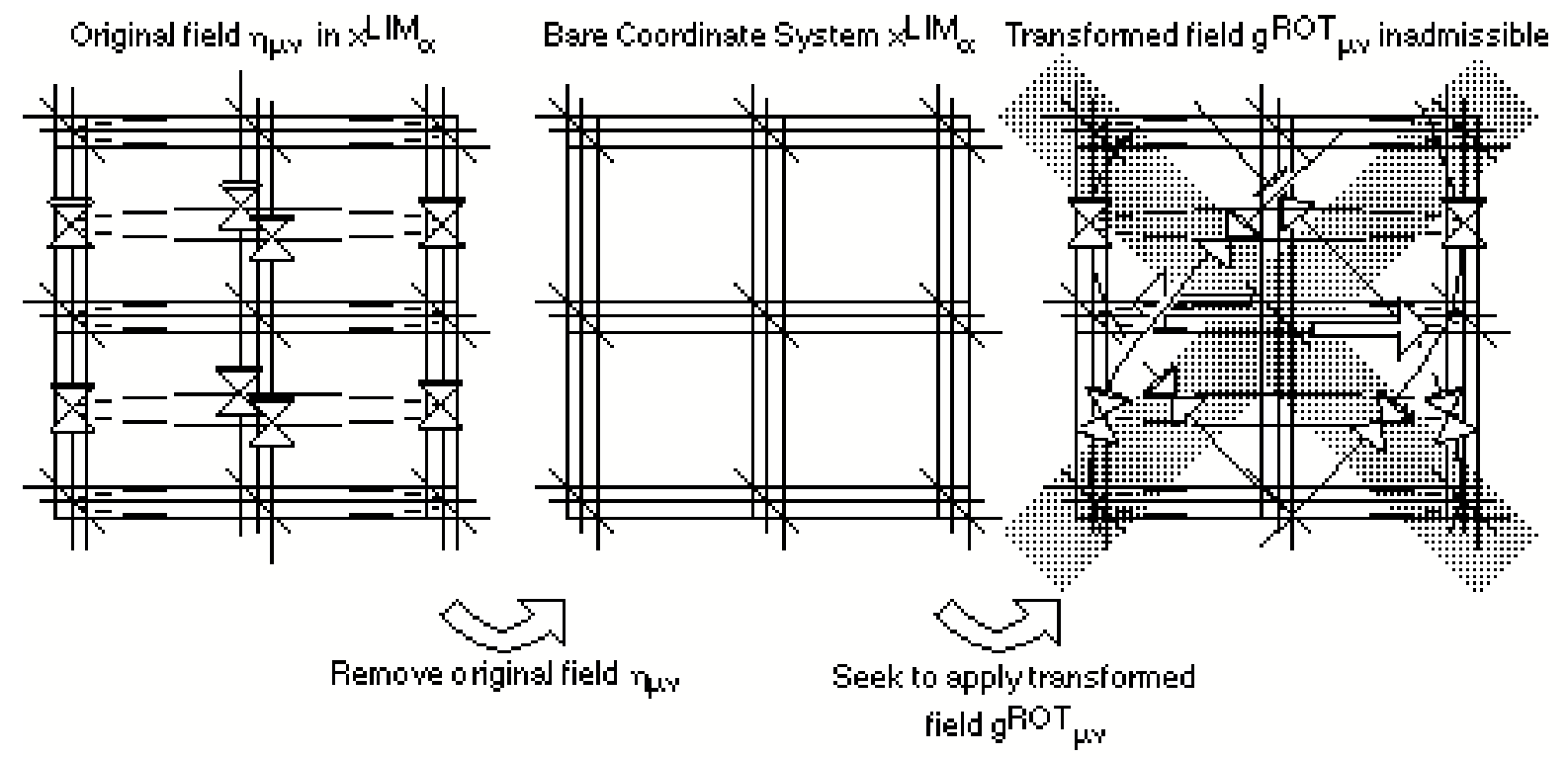

Figure 9. The coordinate system $\mathrm{x}^{\mathrm{LIM}}{ }_{\alpha}$ will not admit the rotation field $\mathrm{g}^{\mathrm{ROT}}{ }_{\alpha}$

During the years of his "Entwurf" theory, Einstein never recognized that his hole argument depended upon the perilous presumption of the independent reality of the coordinate systems. It is an essential part of the present conjecture that Einstein was unaware, correspondingly, that his manipulations depend upon the presumption of the independent reality of the coordinate system $\mathrm{x}^{\mathrm{LIM}}{ }_{\alpha}$. Again, Einstein was so hasty in the earlier presentations of his hole argument that it was unclear whether they used active or passive transformations. Presumably this reflected a lack of attention in distinguishing the two types of transformations. We suppose a similar lack of attention in deciding whether transformations of (11) should be understood actively or passively. 


\title{
6.2 The Anathema of Absolute Coordinate Systems
}

\author{
A fundamental goal of Einstein's work was to find a theory free of the \\ inertial systems of special relativity, which were absolute in their \\ failure to admit the rotation field (13).
}

The failure of $x^{\mathrm{LIM}}{ }_{\alpha}$ to admit the rotation field $\mathrm{g}^{\mathrm{ROT}}{ }_{\mu \nu}$ would have been of the most serious concern to

Einstein. For it showed him that his theory harbored coordinate systems whose properties were routinely decried by him. The coordinate systems $x^{\mathrm{LIM}}{ }_{\alpha}$ would admit the special relativistic metric $\eta_{\mu \nu}$ but it would not admit the rotation field $\mathrm{g}^{\mathrm{ROT}}{ }_{\mu \nu}$. That is, the coordinate systems $\mathrm{x}^{\mathrm{LIM}}{ }_{\alpha}$ behaved just like the inertial systems of special relativity that Einstein was so determined to eradicate. As he explained at the time of the "Entwurf" theory (Einstein 1914a, p.176; translation from Beck, 1996, p. 282) 26

The theory presently called "the theory of relativity" [special relativity] is based on the assumption that there are somehow preexisting "privileged" reference systems $\mathrm{K}$ with respect to which the laws of nature take on an especially simple form, even though one raises in vain the question of what could bring about the privilegings of these reference systems $\mathrm{K}$ as compared with other (e.g., "rotating") reference systems K'. This constitutes, in my opinion, a serious deficiency of this theory.

These inertial systems, as Einstein explained in his text (Einstein, 1922, p.55) supplied special relativity with the absolute elements that he would seek to eliminate in the general theory of relativity. ${ }^{27}$

\footnotetext{
${ }^{26}$ See also Einstein (1913, p.1260).

${ }^{27}$ I have used ellipses liberally in the quote to bring to the fore the aspects of present importance. The complete passage reads:
}

"All of the previous considerations have been based upon the assumption that all inertial systems are equivalent for the description of physical phenomena, but that they are preferred, for the formulation of the laws of nature, to spaces of reference in a different state of motion. We can think of no cause for this preference for definite states of motion to all others, according to our previous considerations, either in the perceptible bodies or in the concept of motion; on the contrary, it must be regarded as an independent property of the space-time continuum. The principle of inertia, in particular, seems to compel us to ascribe physically objective properties to the space-time continuum. 
[Special relativity is based on]...the assumption that all inertial systems are ... preferred, for the formulation of the laws of nature, to spaces of reference in a different state of motion. ... this preference for definite states of motion... must be regarded as an independent property of the spacetime continuum. The principle of inertia, in particular, seems to compel us to ascribe physically objective properties to the space-time continuum. ... from the standpoint of the special theory of relativity we must say, continuum spatii et temporis est absolutum.

In 1913 it would appear to Einstein that the inertial systems of special relativity and now also the coordinate systems ${ }_{x}{ }^{\mathrm{LIM}}{ }_{\alpha}$ endow their spacetimes with certain preferred or absolute motions. These are defined by the natural ${ }^{28}$ straights of either coordinate system, as shown in Figure 10. The trajectories of free bodies are preordained to follow these straights; they may not be curved. Once ${ }^{\mathrm{LIM}}{ }_{\alpha}$ is admitted into the theory, its spacetime will not admit a rotation field $\mathrm{g}^{\mathrm{ROT}}{ }_{\mu \nu}$ in relation to which $\mathrm{x}^{\mathrm{LIM}}{ }_{\alpha}$ would take on the character of a rotating reference system.

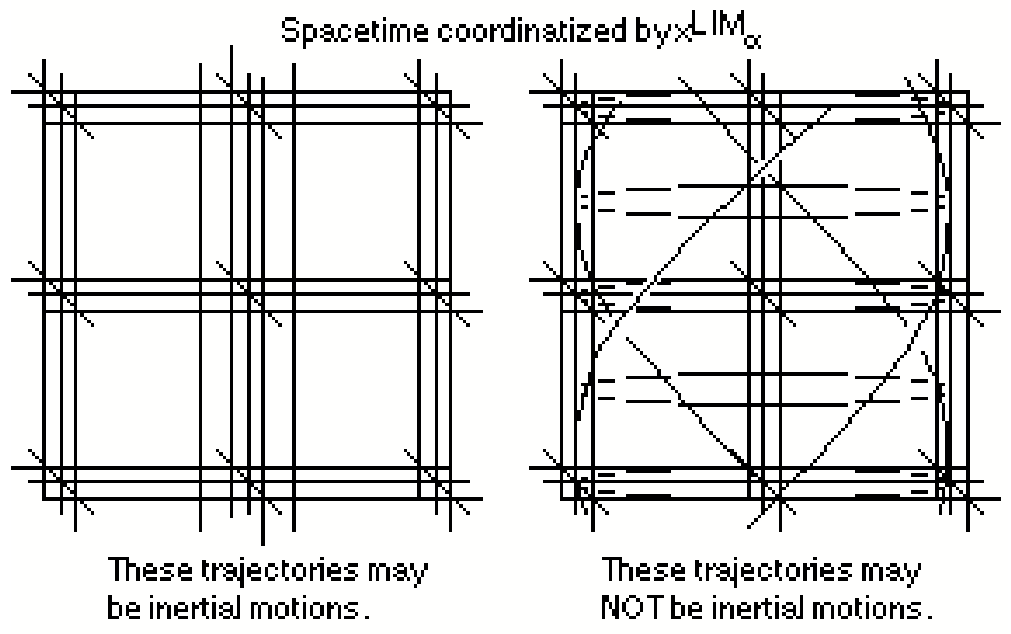

Figure 10. The Coordinate System ${ }^{\mathrm{LIM}}{ }_{\alpha}$ Endows Spacetime With Absolute Properties

Just as it was consistent from the Newtonian standpoint to make both the statements tempus est absolutum, spatium est absolutum, so from the standpoint of the special theory of relativity we must say, continuum spatii et temporis est absolutum. In this latter statement, absolutum means not only 'physically real,' but also 'independent in its physical properties, having a physical effect, but not itself influenced by physical conditions.'"

${ }^{28}$ In a coordinate system $\left(\mathrm{x}_{1}, \mathrm{x}_{2}, \mathrm{x}_{3}, \mathrm{x}_{4}\right)$, these are defined as the curves that satisfy $\mathrm{x}_{4}=\mathrm{A}_{\mathrm{i}} \mathrm{x}_{\mathrm{i}}+\mathrm{B}_{\mathrm{i}}$ for constants $\mathrm{A}_{\mathrm{i}}$ and $B_{i}$ where $i=1,2,3$, where $A_{1}^{2}+A_{2}^{2}+A_{3}^{2}<1$. 
Promising as the gravitation tensor $\mathrm{T}^{\mathrm{x}}{ }_{\mathrm{il}}$ seemed, it appeared inadmissible if Einstein's program of the elimination of absolutes was to succeed. For the if the theory built around this gravitation tensor was to yield the correct Newtonian limit, it was at the cost of introducing exactly such absolute element in the form of the limiting coordinate systems $x^{\mathrm{LIM}}{ }^{29}{ }^{29}$

\subsection{The Theta Condition}

This conjecture explains why Einstein's next step in the Zurich Notebook is to seek to replace the coordinate condition (11) by another stated as a covariance condition contrived to admit rotation

transformations (12)

The conjecture now also explains the calculations to which Einstein turns on the page following, p. $45 / 36$

23L. Having just been thwarted by a coordinate condition of insufficient covariance, he decided to prevent another such failure by defining the coordinate condition from the start as a covariance requirement that had sufficient covariance for his purposes. So he stipulated a restriction to a class of coordinate systems within which the quantity $\theta_{\mathrm{i} \kappa \lambda}$ transforms as a tensor, where

${ }^{29}$ For completeness I note how this conclusion would err in Einstein's later view. In the construction shown in Figure 9, we incorrectly suggest that we seek to apply the two fields $\eta_{\mu \nu}$ and $\mathrm{g}^{\mathrm{ROT}}{ }_{\mu \nu}$ to the same coordinate system. The construction fails because of the illicit intermediate stage in which a bare coordinate system is still supposed to label the same events. There are coordinate systems $\mathrm{x}^{\mathrm{LIM}}{ }_{\alpha}$ compatible with the rotation field $\mathrm{g}^{\mathrm{ROT}}{ }_{\alpha}$ picked out by (11). But their $\mathrm{x}_{4}$ axes would appear helical if drawn in Figure 9 just like the free fall trajectories of particles in $\mathrm{g}^{\mathrm{ROT}}{ }_{\mu \nu}$. Indeed one of these coordinate systems would be the image under rotation transformation (12) of the coordinate system $x^{\mathrm{LIM}}{ }_{\alpha}$ associated with $\eta_{\mu v}$. The coordinate systems $\mathrm{x}^{\mathrm{LIM}}{ }_{\alpha}$ are able to induce absolute properties onto a spacetime of events only as long as we suppose that they are capable of labeling events independently of the metric fields defined on them. The erroneous view requires that it makes sense to assert counterfactual claims like: "This trajectory designated by this coordinate axis could have been a non-inertial motion if there were a different metric field." If removal of the metric field deprives a coordinate system of its ability to designate these trajectories, then the counterfactual loses its meaning. 


$$
\vartheta_{i \kappa \lambda}=\frac{1}{2}\left(\frac{\partial g_{i \kappa}}{\partial x_{\lambda}}+\frac{\partial g_{\kappa \lambda}}{\partial x_{i}}+\frac{\partial g_{\lambda i}}{\partial x_{\kappa}}\right)
$$

His goal is clearly to replace the coordinate condition (11); he writes in the middle of his calculations that "[condition (11)] is not needed." It appears from calculations on other pages that Einstein designed his new coordinate condition to embrace the rotation transformations (12). He failed in this last goal, but only just. ${ }^{30}$

If Einstein believed that the covariance of his theory is restricted to that of the coordinate condition he imposes for recovery of the Newtonian limit, then he gains nothing in limiting the use of the coordinate condition to that special case of the Newtonian limit. He might as well impose the condition universally. That is, he might as well use it as what we have described as a "coordinate restriction" in Section 4.2. His gravitation tensor, to be used universally, will then be whatever remains of the tensor he starts with, after the coordinate restriction has been applied. This seems to be Einstein's purpose. On p. 45/ 36 23L, he takes the gravitation tensor $\mathrm{T}^{\mathrm{x}}{ }_{\mathrm{il}}$ and adds and subtracts terms in $\theta_{\mathrm{i} \kappa} \lambda$ until he arrives at a gravitation tensor of the form required by (8) and which is also by construction a tensor under unimodular transformation for which $\theta_{\mathrm{i} \kappa \lambda}$ transforms as a tensor. That he freely adds these terms shows that his interest lies just in the final result and its covariance, for that final result will not be a quantity to which $\mathrm{T}^{\mathrm{x}}{ }_{\mathrm{il}}$ reduces to in the restricted set of coordinate systems.

If these last transformations had included the rotation transformations (12) then Einstein would have succeeded where he had failed on the previous page, in finding a gravitational field equation, covariant under rotation transformations and of form (8). But they did not include them and, apparently for this reason, the proposal of the theta condition was abandoned. Nonetheless, the introduction of this theta condition on p.45/3623L in an

\footnotetext{
${ }^{30}$ If the coordinate condition admits these rotation transformations, then it must admit transformations between the special relativistic metric $\eta_{\mu \nu}$ and the rotation field $\mathrm{g}^{\mathrm{ROT}}{ }_{\mu \nu}$. Since $\eta_{\mu \nu}$ has constant coefficients, we have $\theta_{\mathrm{i} \kappa} \lambda=0$ for it. If $\theta_{i \kappa \lambda}$ transforms tensorially under (12), then we must find that $\theta_{\mathrm{i} \kappa} \lambda=0$ also for $\mathrm{g}^{\mathrm{ROT}}{ }_{\mu \nu}$. On pp.7-8/3 $642 \mathrm{~L}-$ $42 \mathrm{R}$ Einstein is apparently checking this expectation when he seeks all fields compatible with the conditions $\theta_{\mathrm{i} \kappa} \lambda=0$, with metrics of unit determinant and $\frac{\partial g_{i k}}{\partial x_{4}}=0$. (These last two conditions are satisfied by g ${ }^{\text {ROT }}{ }_{\mu v}$.) His
} expectations are almost vindicated. The solution class includes a metric whose coefficients in the covariant form equals the coefficients of the metric $\mathrm{g}^{\mathrm{ROT}}{ }_{\mu \nu}$ in its contravariant form. This is close, but it is not the metric $\mathrm{g}^{\mathrm{ROT}}{ }_{\mu \nu}$. 
ingenious response to the difficulties Einstein believed he encountered on with the gravitation tensor $\mathrm{T}^{\mathrm{x}_{i 1}}$ on the preceding page p.44/ $3622 \mathrm{R}$.

\title{
6.4 The Structure and Fate of the Entwurf Theory
}

\author{
The conjecture explains why Einstein was uninterested in finding the \\ generally covariant gravitational field equations which reduce to his \\ "Entwurf" equations. It also suggests that recognition of the \\ admissibility of the gravitation tensor $T^{x}$ il and rejection of the hole \\ argument could come at the same time since they are based on the
}

same error.

The conjecture explains why Einstein set up and developed the Entwurf theory as he did and illuminates his return to general covariance. It suggests something quite general about the way Einstein would have sought to build his gravitation theory. According to the conjecture, as noted in Section 6.3 , the covariance of the theory as a whole is limited to the covariance of the coordinate condition used to recover the Newtonian limit. The coordinate condition asserts the existence of coordinate systems $\mathrm{x}^{\mathrm{LIM}}{ }_{\alpha}$ which in turn attribute absolute properties to spacetime, whether we are in the domain of the Newtonian limit or not. Thus Einstein purchases no additional covariance for his theory if he considers his gravitational field equations before they are reduced by the coordinate condition used to recover the Newtonian limit. He may as well work with the field equations after they have been reduced to the form (8).

This turns out to be just what Einstein does. The gravitational field equations published in the "Entwurf" theory have the form (8). From remarks in several places (for example Einstein, 1914a, pp.177-78), we know that he was sure that the "Entwurf" gravitational field equations were reduced forms of some unknown generally covariant equations, but he dismissed efforts to discover them as "premature" in the "Entwurf" paper (Einstein and Grossmann, 1913, I.§5). (His attitude had hardened after he found the hole argument; then he dismisses these efforts as "of no special interest." (Einstein, 1914a, p.179).) That these efforts should be dismissed so quickly right from the first publication of the "Entwurf" theory is inexplicable on the modern view. For finding these equations would immediately dispel the uncertainty surrounding his theory: he did not know the extent of the covariance of the equation of the "Entwurf" theory. He could then use those generally covariant equations as his field equations and 
thereby present the world a theory that was manifestly generally covariant. Under the conjecture, however, his lack of interest is readily explicable. Finding those generally covariant equations would not allow him to add any covariance to his theory.

The conjecture allows us to see the connections between the events comprising Einstein's return to general covariance late in 1915. In our documentary records, that return began in earnest with a letter to Erwin Freundlich of September 30, 1915, (Papers, Vol. 8A, Doc. 123) in which an alarmed and weary Einstein reported his horror at discovering that his "Entwurf" equations were not covariant under the rotation transformation (12). In his communication of November 4 to the Prussian Academy, Einstein (1915) reports his return to the search for generally covariant gravitational field equations and that his choice of gravitation tensor is $\mathrm{T}^{\mathrm{x}}{ }_{\mathrm{il}}$.

We can now readily see how they could be connected. Their common feature is rotational covariance, that is, covariance under (12): Einstein had just found that his "Entwurf" equations lack it; he had rejected $\mathrm{T}^{\mathrm{x}}{ }_{\mathrm{il}}$ because the associated coordinate condition (11) lacked it. We can guess many scenarios that lead from the discovery of the lack of covariance of the "Entwurf" equations to the readmission of the gravitation tensor $\mathrm{T}^{\mathrm{x}}$. For example, ${ }^{31}$ Einstein was shocked to find that even the "Entwurf" gravitational field equations lacked covariance under rotation transformations (12). That he mistakenly thought these equations unique made the problem all the more acute. It would be natural in that circumstance to review the other candidate gravitation tensors from his earlier investigations that were covariant under transformation (12). They were the Ricci tensor and $\mathrm{T}^{\mathrm{x}}{ }_{\mathrm{il}}$. The Ricci tensor remained inadmissible because of its incompatibility with the flatness of weak, static fields. The tensor $\mathrm{T}^{\mathrm{x}_{i 1}}$ did have the requisite covariance; it failed only when the associated coordinate condition (11) was considered. A devastated Einstein, now willing to think things through once again from the start, might well now see that his reasons for rejecting $\mathrm{T}^{\mathrm{x}_{i l}}$ were based on the error of according the coordinate systems $\mathrm{x}^{\mathrm{LIM}}{ }_{\alpha}$ an existence independent of the metric field. The result would be his November 4 communication of the gravitation tensor $\mathrm{T}^{\mathrm{x}}{ }_{\mathrm{il}}$ to the Prussian

\footnotetext{
${ }^{31}$ With the repertoire supplied by the conjecture, finding other scenarios is merely a challenge to one's ingenuity. Einstein may instead have begun by deciding that he must return to general covariance and so reappraised the hole argument, his public objection to such a return. With the error of that argument found, the readmission of tensor $\mathrm{T}^{\mathrm{x}} \mathrm{il}$ is now possible since its rejection was based on that same error.
} 
Academy. His final choice of the Ricci tensor and then Einstein tensor would only come in later communications that month after he recognized his other error of requiring the spatial flatness of weak, static fields.

But what of the hole argument? By November 4, Einstein could not have thought it succeeded in showing that a generally covariant theory was physically uninteresting for he was urging acceptance of a theory of near general covariance. One possibility is that Einstein had merely decided it must be flawed and that he would seek that flaw once the more pressing problem of finding generally covariant gravitational field equations had been solved. The conjecture suggests another possibility. According to it, the error of the hole argument and the error of the rejection of $\mathrm{T}^{\mathrm{x}_{\mathrm{il}}}$ are the same--improperly according a reality to coordinate systems independently of the metric fields defined on them. So once he located the error in one he had automatically found the error in the other. We might well understand that he would delay formulating a polished, public statement of the error of the hole argument until after November 1915. The real work was the completion of the theory by finding generally covariant equations, not drawing further attention to his earlier errors. ${ }^{32}$

There is scant evidence directly connecting the rejection of the hole argument and Einstein's discovery of the "Entwurf" theory's lack of rotational covariance. Most striking is a remark made to de Sitter in a letter of January 23, 1917 (Papers, Vol. 8A, Doc. 290). He reflected on two errors in his review, Einstein (1914): the hole argument and another defective consideration. "I noticed my mistakes from that time," he recalled, " when I calculated directly that my field equations of that time were not satisfied in a rotating system in a Galilean space." Without the

\footnotetext{
${ }^{32}$ Einstein appears to have delayed informing his correspondents of the error of the hole argument and avoided mentioning the argument directly in print thereafter. In the surviving correspondence, the first explanation comes in the letter to Ehrenfest of December 26, 1915 (Papers, Vol. 8A, Doc. 173), which advances the point-coincidence argument. That argument is published in his review article the following year (Einstein, 1916, §3), but the argument is presented as one favoring general covariance without any indication that it is his own response to the hole argument. See Howard and Norton $(1993, \S 7)$ for the suggestion that Einstein may have chosen to formulate his response to the hole argument in terms of point-coincidences upon the unacknowledged inspiration of a paper by Kretschmann (1915) from December 1915.
} 
conjecture of this paper, it is hard to see why this calculation in a rotating system would have any direct bearing on the hole argument. With the conjecture, the connection is direct. 


\section{Conclusion}

The present conjecture resolves outstanding puzzles in our history

without the need to conjecture a new error by Einstein. The hole

argument proves to capture precisely the essential obstacle separating

Einstein from general covariance, although prior to 1915 he

misidentified the obstacle it revealed .

The case for the conjecture is necessarily indirect. Unlike Einstein's error concerning the spatial flatness of weak, static fields, we do not have a direct, written admissions by Einstein that he committed it. However some such error must be conjectured to complete our account of Einstein's search for his gravitational field equations. The other candidate explanation is the supposition that Einstein was just unaware of the modern use of coordinate conditions, even though he had the mathematical manipulations associated with them in his notebook. I do not believe he had this unawareness for reasons sketched in Section 4.2. The final decision depends considerably on a question of plausibility. Do we lean towards an obtuse Einstein, who persistently overlooks the obvious? Or do we prefer an Einstein able to commit an error of Byzantine sophistication? In the absence of good evidence for the former error, I choose the latter. The resulting account just takes the one other fundamental error that Einstein later freely admitted, the error of the hole argument. It asks after the consequences if that error were committed also months earlier in another context, that of the recovery of the Newtonian limit from candidate gravitational field equations.

The result is a compelling account of how Einstein came to abandon the search for generally covariant gravitational field equations in 1913. It was not just an oversight on Einstein's part. Very formidable obstacles separated him from the final, generally covariant gravitational field equations of 1915 . He had to abandon his presumption of the spatial flatness of weak, static fields. Yet he had multiple items of independent evidence for it: it was suggested by his principle of equivalence, by the equations of motion of a particle in free fall and by the simplest form naturally taken by the gravitational equations in the weak field. Even if he could have seen past this problem, I now conjecture that a deeper misconception assured his failure. It lay buried beneath his conscious 
awareness, but misdirected fatally his attempts to use coordinate conditions.. As long as he tacitly attributed an independent reality to spacetime coordinate systems, he must demand that the covariance of his theory be limited to the covariance of the coordinate condition used to recover the Newtonian limit from his gravitational field equations. Not even Einstein could be expected to find gravitational field equations that were otherwise admissible and associated with a coordinate condition of sufficiently broad covariance to support a generalized principle of relativity.

These were obstacles worthy of an Einstein and able to delay him for over two years in his struggle with his general theory of relativity. The hole argument proves to be more than an afterthought used to explain a decision already taken for other reasons. This argument, which Einstein repeatedly offered to explain the inadmissibility of generally covariant gravitational field equations, turns out to depend essentially on one of the two major obstacles recounted here--although Einstein misdiagnosed the import of the argument prior to 1915 . We now see that it does not force us to abandon general covariance; rather its shows us we must abandon the notion that coordinate systems have a reality independent of the metric fields defined on them. Until Einstein did that, his quest for a generally covariant theory could only fail. 


\section{References}

Beck, Anna (1996) Translation. The Collected Papers of Albert Einstein. Vol. 4 The Swiss Years: Writings, 1912-

1914. Don Howard, consultant. Princeton: Princeton University Press.

Earman, John and Janssen, Michel (1993) "Einstein's Explanation of the Motion of Mercury's Perihelion" in John

Earman,Michel Janssen and John D. Norton The Attraction of Gravitation: New Studies in the History of

General Relativity: Einstein Studies, Volume 5. Boston: Birkhäuser.

Einstein, Albert (1907) "Über das Relativitätsprinzip und die aus demselben gezogenen Folgerungen," $\underline{\text { Jahrbuch der }}$

Radioaktivität und Elektronik, 4(1907), 411-462; $\underline{5}(1908), 98-99$.

Einstein, Albert (1911): "Über den Einfluss der Schwerkraft auf die Ausbreitung des Lichtes," Annalen der Physik,

$\underline{35}, 898-908$; translated as "On the Influence of Gravitation on the Propagation of Light, " pp.99-108 in

H.A.Lorentz et al., The Principle of Relativity. Dover, 1952.

Einstein, Albert (1912a): "Lichtgeschwindigkeit und Statik des Gravitationsfeldes," Annalen der Physik, 38, 355-69.

Einstein, Albert (1912b): "Zur Theorie des Statischen Gravitationsfeldes," Annalen der Physik, $\underline{38}, 443-58$.

Einstein, Albert (1913) "Zum gegenwärtigen Stande des Gravitationsproblems," Physikalische Zeitschrift, 14,

pp.1249-1262.

Einstein, Albert (1914): "Die formale Grundlage der allgemeinen Relativitätstheorie," Königlich Preussische Akademie der Wissenschaften (Berlin). Sitzungsberichte., pp.1030-1085.

Einstein, Albert (1914a) "Prinzipielles zur verallgemeinerten Relativistätstheorie," Physikalische Zeitschrift 15, 17680.

Einstein, Albert (1915) "Zur allgemeinen Relativitätstheorie," Königlich Preussische Akademie der Wissenschaften (Berlin). Sitzungsberichte. 1915, pp.778-86.

Einstein, Albert (1915a) "Erklärung der Perihelbewegung des Merkur aus der allgemeinen Relativitätstheorie," Königlich Preussische Akademie der Wissenschaften (Berlin). Sitzungsberichte. 1915, pp.831-39. 
Einstein, A. (1916) "Die Grundlage der allgemeinen Relativitätstheorie," Annalen der Physik, 49, 769-822; translated without p.769 as "The Foundation of the General Theory of Relativity," pp. 111-164 in H.A.Lorentz et al., The Principle of Relativity. Dover, 1952.

Einstein, Albert (1922) The Meaning of Relativity. 5th ed. Princeton: Princeton Univ. Press, 1974.

Einstein, Albert (1954) Relativity: the Special and the General Theory. Trans. R. W. Lawson. 15th rev. ed. London: Methuen, 1977.

Einstein, Albert and Grossmann, Marcel (1913): Entwurf einer verallgemeinerten Relativitätstheorie und einer Theorie der Gravitation. Leibzig: B.G.Teubner (separatum); with addendum by Einstein in Zeitschrift für Mathematik und Physik, $\underline{63}$ (1914), pp.225-61. (ㄹpers, Vol. 4)

Einstein, Albert and Rosen, Nathan (1935) "The Particle Problem in the General Theory of Relativity," Physical Review, $\underline{48}$, pp.73-77.

Howard Don and Norton John D. (1993), "Out of the Labyrinth? Einstein, Hertz and the Göttingen Answer to the Hole Argument," pp. 30-62 in John Earman, Michel Janssen, John D. Norton (eds.) The Attraction of Gravitation: New Studies in History of General Relativity Boston: Birkhäuser.

Janssen, Michel (1999) "Rotation as the Nemesis of Einstein's 'Entwurf' Theory," in H. Goenner, J. Renn, J. Ritter and T. Sauer (eds.) The Expanding Worlds of General Relativity: Einstein Studies, Vol. 7 , Boston: Birkhäuser, 1999, pp. 127-157.

Klein, Martin J. et al. (1993) The Collected Papers of Albert Einstein. Volume 5. The Swiss Years: Correspondence, 1902-1014. Princeton: Princeton University Press. ("Papers, Vol. 5")

Klein, Martin J. et al. (eds.) (1995) The Collected Papers of Albert Einstein. Volume 4. The Swiss Years: Writing, 1912-1914. Princeton: Princeton Univ. Press. ("Papers, Vol.4")

Kretschmann, Erich (1915) "Über die prinzipielle Bestimmbarkeit der berechtigten Bezugssystemen beliebiger Relativitätstheorien." Annalen der Physik 48: 907-982

Norton, John D.(1983)"Einstein's Struggle with General Covariance," Rivista di Storia della Scienza, 2 (1985), pp. 191-205. 
Norton, John D. (1984) "How Einstein found his Field Equations: 1912-1915," Historical Studies in the Physical

Sciences, 14, 253-316; reprinted in Don Howard and John Stachel (eds.) Einstein and the History of General Relativity: Einstein Studies, Vol. 1 Boston: Birkhäuser, 1989, pp.101-159.

Norton, John D. (1985): "What was Einstein's Principle of Equivalence?" Studies in History and Philosophy of

Science, 16, 203-246; reprinted in Don Howard and John Stachel (eds.) Einstein and the History of General

Relativity: Einstein Studies, Vol. 1 Boston: Birkhäuser, 1989, pp.5-47.

Norton, John D. (1986)"Einstein's Struggle with General Covariance," in R. Ruffini (ed.) Proceedings of the Fourth Marcel Grossmann Meeting on General Relativity, (Elsevier Science Publishers B.V.), pp. 1837-48.

Norton, John D.(1987): "Einstein, the Hole Argument and the Reality of Space," pp. 153-188 in John Forge (ed.) Measurement, Realism and Objectivity. Reidel.

Norton, John D. (1989) "Coordinates and Covariance: Einstein's view of spacetime and the modern view,"

Foundations of Physics, 19 , 1215-1263.

Norton, John D. (1992) "Einstein, Nordström and the Early Demise of Scalar, Lorentz-Covariant Theories of Gravitation," Archive for the History of Exact Sciences, 45, pp. 17-94.

Norton, John D. (1992a) "The Physical Content of General Covariance" in J. Eisenstaedt and A. Kox eds., Studies in the History of General Relativity: Einstein Studies, Vol.3, Boston: Birkhauser.

Norton, John D. (1993), "General Covariance and the Foundations of General Relativity: Eight Decades of Disute," Reports on Progress in Physics, $\underline{56}$, pp. 791-858.

Norton, John D. (forthcoming) "What was Einstein's Fateful Prejudice?" in Renn, Sauer et al. (forthcoming).

Renn, Jürgen (forthcoming) "Progress in a Loop; General Relativity as a Triumph of the 'Entwurf' Theory” in Renn, Sauer et al. (forthcoming).

Renn, Jürgen and Sauer, Tilman (1999) "Heuristics and Mathematical Representation in Einstein's Search for a Gravitational Field Equation," in H. Goenner, J. Renn, J. Ritter and T. Sauer (eds.) The Expanding Worlds of General Relativity: Einstein Studies, Vol. 7 , Boston: Birkhäuser, 1999, pp. 87-125.

Renn, Jürgen; Sauer,Tilman et al. (forthcoming) A Commentary on Einstein's Zurich Notebook. 
Schulmann, Robert; Kox, A. J.; Janssen, Michel and Illy, József (eds.) (1998) The Collected Papers of Albert Einstein. Volume 8. The Berlin Years: Correspondence, 1914-1918. Part A: 1914-1917. Princeton: Princeton Univ. Press. ("Papers, Vol.8A").

Speziali, Pierre, ed., (1972) Albert Einstein--Michele Besso: Correspondance 1903-1955 Paris: Hermann.

Stachel, John (1980): "Einstein's Search for General Covariance," paper read at the Ninth International Conference on General Relativity and Gravitation, Jena; printed in Don Howard and John Stachel (eds.) Einstein and the History of General Relativity: Einstein Studies, Vol. 1 (Boston: Birkhäuser, 1989) pp.63-100. 